\title{
Fuel molecular structure effect on autoignition of highly branched iso-alkanes at low-to-intermediate temperatures: iso-octane versus iso-dodecane
}

\author{
Ruozhou Fang ${ }^{1}$, Goutham Kukkadapu ${ }^{2 *}$, Mengyuan Wang ${ }^{1 *}$, Scott W. Wagnon ${ }^{2}$, \\ Kuiwen Zhang ${ }^{2}$, Marco Mehl², Charles K. Westbrook ${ }^{2}$, William J. Pitz ${ }^{2}$, Chih-Jen Sung ${ }^{1}$ \\ ${ }^{1}$ University of Connecticut, Department of Mechanical Engineering, Storrs, CT 06269, USA \\ ${ }^{2}$ Lawrence Livermore National Laboratory, Livermore, CA 94551, USA
}

KEYWORDS: iso-octane, iso-dodecane, autoignition, rapid compression machine, low temperature chemistry

Corresponding Authors:

Mengyuan Wang

Phone: 860-486-2492

Email: mengyuan.wang@uconn.edu

Goutham Kukkadapu

Phone: 925-422-1489

Email: kukkadapu1@1lnl.gov

Submitted for publication in Combustion and Flame

December 23, 2019 


\title{
Fuel molecular structure effect on autoignition of highly branched iso-alkanes at low-to-intermediate temperatures: iso-octane versus iso-dodecane
}

\author{
Ruozhou Fang1, Goutham Kukkadapu ${ }^{2 *}$, Mengyuan Wang ${ }^{1 *}$, Scott W. Wagnon ${ }^{2}$, \\ Kuiwen Zhang'2, Marco Mehl'2, Charles K. Westbrook ${ }^{2}$, William J. Pitz², Chih-Jen Sung ${ }^{1}$ \\ ${ }^{1}$ Department of Mechanical Engineering, University of Connecticut, Storrs, CT, USA \\ ${ }^{2}$ Lawrence Livermore National Laboratory, Livermore, CA, USA \\ *Corresponding Authors: mengyuan.wang@uconn.edu, kukkadapu1@1lnl.gov
}

\begin{abstract}
Highly branched iso-alkanes are an important class of hydrocarbons found in conventional petroleumderived and alternative renewable fuels used for combustion applications. Recognizing that chemical kinetics for most of these iso-alkanes, especially at low-to-intermediate temperatures, has not been well studied, an experimental and modeling investigation of two selected iso-alkanes, iso-octane (2,2,4trimethylpentane, iC8) and iso-dodecane (2,2,4,6,6-pentamethylheptane, iC12), is conducted to understand the fuel molecular structure effect on their autoignition characteristics. Using a rapid compression machine (RCM), the ignition responses of $\mathrm{iC} 8$ and $\mathrm{iC} 12$ at varying pressures, temperatures, and equivalence ratios are characterized and compared. The newly-acquired experimental ignition delay times have been compared with the literature RCM and shock tube data, demonstrating the complementary nature of the current dataset. Further comparison of the experimental pressure traces and ignition delay times illustrates the reactivity crossover between iC8 and iC12. Namely, there exists a temperature window in the negative temperature coefficient regime within which $\mathrm{iC} 12$ is less reactive than $\mathrm{iC} 8$, but $\mathrm{iC} 12$ becomes more reactive outside this temperature window. Furthermore, a chemical kinetic model of iso-alkanes including both $\mathrm{iC} 8$ and iC12 is developed. Simulated results using this model are then compared to the experimental data obtained in this study and available in the literature, showing its ability to predict the experimental trends. Chemical kinetic analyses have also been conducted to identify the important reaction pathways controlling autoignition at varying conditions, and to elucidate the underlying mechanism leading to different reactivity trends between $\mathrm{iC} 8$ and $\mathrm{iC} 12$.
\end{abstract}




\section{Introduction}

Modeling conventional and alternative transportation fuels is challenging because those real fuels are composed of hundreds to thousands of compounds. As it is nearly impossible to consider all constituents present in transportation fuels due to huge model size, one promising approach of reducing model size is to group the fuel components into structural classes, i.e., $n$-alkanes, iso-alkanes, aromatics, etc., and to select a few representative pure components from each structural class to formulate a more compact surrogate fuel mixture matching certain properties of the target real fuel [1-5]. As such, studying the combustion characteristics as well as chemical kinetics of each structural class with increasing molecular size can enhance fundamental understanding of the governing chemistry of each structural class and further facilitate the surrogate fuel formulation and model development.

There have been limited studies that systematically investigated chemical kinetics of a specific structural class with increasing molecular sizes. Notably, Westbrook et al. [3] developed a detailed reaction mechanism for combustion of $n$-alkanes from $C_{8}$ to $C_{16}$, while Sarathy et al. [4] studied the oxidation of 2-methylalkanes, lightly-branched alkanes, from $\mathrm{C}_{7}$ to $\mathrm{C}_{20}$. Although iso-alkanes are considered as one of the backbone structural classes for surrogate fuel modeling, its combustion characteristics as well as chemical kinetics are still not well understood, especially in the low-tointermediate temperatures regime. As the low temperature combustion concept has received tremendous attention due to its substantially reduced pollutant emissions [6,7] and high thermodynamic efficiency [8], it is of fundamental interest to explore the autoignition characteristics of various iso-alkanes at lowto-intermediate temperatures. In this study, two representative highly-branched iso-alkanes, iso-octane (2,2,4-trimethylpentane, iC8) and iso-dodecane (2,2,4,6,6-pentamethylheptane, iC12), are selected for highlighting the fuel molecular structure effect on autoignition under engine relevant conditions. Figure 1 shows the molecular structures of $\mathrm{iC} 8$ and $\mathrm{iC} 12$ investigated herein.

Fundamental combustion experiments investigating the autoignition characteristics of iC8 have been extensively conducted over the past few decades, including shock tube (ST) studies at high-to- 
intermediate temperatures [9-16] and rapid compression machine (RCM) studies at low-to-intermediate temperatures [17-25]. Griffiths et al. [17] reported the autoignition characteristics of $n$-alkanes from $\mathrm{C}_{5}$ to $\mathrm{C}_{8}$, two iso-alkanes (iso-butane and $\mathrm{iC} 8$ ), and toluene at stoichiometric condition in air over the temperature range of $600-950 \mathrm{~K}$ and pressures up to $0.9 \mathrm{MPa}$. Considering it as a component of primary reference fuels, autoignition of iC8 at temperatures up to $1000 \mathrm{~K}$ was investigated in [18-20]. He et al. $[21,22]$ studied the autoignition of $\mathrm{iC} 8 / \mathrm{O}_{2} / \mathrm{N}_{2} / \mathrm{Ar}$ mixtures at equivalence ratios from fuel-lean to stoichiometric, pressures up to $23 \mathrm{~atm}$, and temperatures of 943-1027 K. Walton et al. [23] investigated ignition phenomena of iC8/air mixtures at equivalence ratios of $0.2-1.98$, temperatures of 903-1020 K, and pressures of 8.7-16.6 atm. Mittal and Sung [24] conducted autoignition tests using stoichiometric $\mathrm{iC} 8 / \mathrm{O}_{2} /$ inert gas mixtures at pressures of 13-16 bar and temperatures of 680-880 K. More recently, Atef et al. [25] studied iC8 autoignition in several RCMs from different institutions, with pressures up to 40 atm and temperatures up to $1000 \mathrm{~K}$.

Chemical kinetic models of iC8 have also been developed in many previous studies. At first, the mechanisms including iC8 were either created by automatic generation mechanism package [26] or semi-detailed in nature $[19,27,28]$. A detailed chemical kinetic model of primary reference fuel mixtures was developed by Curran et al. [29] and used to simulate flow reactor experiments on the lean oxidation of iC8 in the intermediate temperature regime at elevated pressures [30]. Based on [29], Curran et al. [31] developed a detailed chemical kinetic model to study the oxidation of iC8 from low to high temperatures. Mehl et al. [32,33] further developed a low temperature reaction mechanism of iC8 for better prediction, but there are still some experimental data at fuel lean conditions that are difficult to match. Most recently, Atef et al. [25] presented an updated chemical kinetic model for iC8 combustion, with thermodynamic data and reaction kinetics being re-assessed based on new thermodynamic group values and newly evaluated rate coefficients from the literature. This updated model was compared against RCM and ST experiments and has shown improved agreement at lower equivalence ratios compared to other literature mechanisms. 
By contrast, limited autoignition data for iC12 have been reported [34-36]. Won et al. [34] measured the reflected shock ignition delay times of iC12 from stoichiometric to fuel-rich at 20 and 40 atm, and compared the iC12 data to those of iC8, iso-cetane (2,2,4,4,6,8,8-heptamethylnonane, iC16), and a 50/50 molar blend of $\mathrm{iC} 8 / \mathrm{iC} 16$, reporting that all the ignition delay times at high-to-intermediate temperatures are similar. Flora et al. [35] further studied the ignition characteristics of a few hydrocarbons proposed as jet fuel surrogate components, including $n$-heptane, $n$-dodecane, $m$-xylene, $n$ dodecane/m-xylene blend (77/23 by volume), and iC12. The shock tube experiments of [35] were conducted at temperatures of 980-1800 K, pressure around 16 atm, and equivalence ratio of 0.5 . Recently, Mao et al. [36] studied iC12 autoignition using RCM and ST with equivalence ratios of 0.5, 1.0, and 1.5, pressures of 15 and 20 bar, and temperatures of 603-1376 K. An iC12 kinetic model was also developed in [36] to predict iC12 total ignition delay times. It is noted that no first-stage ignition delay data of $\mathrm{iC} 12$ was reported in [36], while iC12 is expected to exhibit two-stage ignition response at low temperatures. As the controlling reactions for the first-stage and second-stage ignition are different, it is possible that both simulated first-stage and second-stage ignition delay times are off compared to the experiments while the sum yields a well-predicted total ignition delay time. Providing both firststage and total ignition delay times in RCM datasets allows a more rigorous validation of kinetic models for iC12 and other fuels with two-stage ignition behavior.

In view of the limited autoignition datasets for iC12 at low-to-intermediate temperatures, especially the first-stage ignition delay measurements, RCM experiments over a wide range of conditions are therefore conducted herein to fill this fundamental void. These newly-acquired RCM datasets, along with those reported in [36], are of importance for model validation. Moreover, the comparison of iC8 and iC12 data obtained from the same RCM provides insights into the molecular size/structure effect on autoignition as well as the development of a comprehensive chemical kinetic model for combustion of iso-alkanes from $\mathrm{C}_{8}$ to $\mathrm{C}_{16}$ (or larger). In this investigation, $\mathrm{RCM}$ experiments of iC8/air and iC12/air mixtures at varying pressures $(15,20$, and 30 bar), equivalence ratios $(\phi=0.7,1.0,1.2$, and 2.0$)$, and 
temperatures (600-900 K) are conducted. For some overlapped conditions, the current RCM data are compared with the RCM data of iC8 [25] and iC12 [36] and the ST data of iC8 [14] and iC12 [34,36], in order to demonstrate the consistency of the current RCM results and the literature RCM/ST data. A detailed chemical kinetic model, including low-to-intermediate temperature chemistry for both iC8 and iC12, is also developed. The performance of this chemical kinetic model is validated against the current RCM datasets and the literature data. Furthermore, model-based reaction pathway and sensitivity analyses are performed to identify the key reactions controlling the autoignition of iC8 and iC12, and to delineate the molecular structure effects that lead to different reactivity trends between iC 8 and iC12.

\section{Experimental Specifications}

Measurements of ignition delay times for iC8/air and iC12/air mixtures are conducted in an RCM, consisting of a heated reaction chamber, a piston arrangement, a hydraulic chamber, and a driving tank. The piston arrangement is pneumatically driven and hydraulically stopped, with a specially-designed crevice to suppress the vortex roll-up effect to ensure homogeneity during and after compression. The premixed fuel/air mixture in the cylindrical reaction chamber is compressed by the piston rapidly to reach a specific elevated pressure and temperature at the end of compression (EOC). The reactant mixtures in this study are prepared in a stainless-steel mixing tank, which is connected to the reaction chamber by a manifold. The mixing tank, the manifold, and the reaction chamber are equipped with heaters. A magnetic stirrer is also equipped at the bottom of mixing tank to facilitate preparing a homogeneous mixture. Pressure profiles are considered as the primary diagnostic on the RCM experiments and are recorded by a piezo-electrical pressure transducer (Kistler 6125C-C20) along with a charge amplifier (Kistler 5010B). The EOC temperature and pressure $\left(T_{C}\right.$ and $\left.P_{C}\right)$ can be adjusted independently by varying overall compression ratio, initial pressure $\left(P_{0}\right)$, and initial temperature $\left(T_{0}\right)$. The detailed information about the current RCM can be found in [24,37].

A Python package UConnRCMPy [38] is used for data processing raw experimental pressure traces. The raw experimental pressure traces are first filtered using a first-order Butterworth filter to generate 
filtered pressure traces. The filtered pressure traces are then fed to the data processing block of UConnRCMPy, in which $T_{C}, P_{C}$, and ignition delay times can be determined. The filtered pressure traces are reported herein instead of the raw experimental pressure traces. Figure 2(a) shows the representative experimental pressure trace obtained by the RCM, as well as the definitions of the firststage ignition delay time $\left(\tau_{1}\right)$ and the total ignition delay time $(\tau)$. Namely, $\tau_{1}$ and $\tau$ are defined as the time from the EOC to the respective maximum of the first order time derivative of pressure. At least four consecutive runs are taken for each experimental condition to ensure repeatability, as shown in Fig. 2(b). The typical scatters of $\tau_{1}$ and $\tau$ are less than $15 \%$ of the reported values.

To determine the facility effects (i.e., the heat transfer effect [39]) on the reactive experiment and to confirm that there is no exothermicity during the compression stroke, the corresponding non-reactive experiment is conducted by replacing $\mathrm{O}_{2}$ in the reactive mixture with $\mathrm{N}_{2}$, while keeping the similar specific heat. It is seen from Fig. 2(a) that the pressure trace of the reactive experiment during the compression stroke and prior to the first-stage ignition after reaching the EOC is well captured by the non-reactive counterpart. The $T_{C}$ can be deduced from the measured pressure trace by applying the "adiabatic core hypothesis" [39]. Under this hypothesis, the heat transfer from the reaction chamber only occurs within the thin boundary layer near the wall, while the core region remains adiabatic, and any heat loss during or after the compression stroke is modeled by a change in the modeled volume of the reaction chamber [40]. Therefore, the core region undergoes an isentropic compression during the compression stroke, and the isentropic relation can be applied to calculate $T_{C}$ via:

$$
\int_{T_{0}}^{T_{C}} \frac{\gamma}{\gamma-1} \frac{d T}{T}=\ln \left(\frac{P_{C}}{P_{0}}\right)
$$

where $\gamma$ is the temperature-dependent specific heat ratio. In addition, the Python interface of Cantera 2.3.0 [41] is used to compute $T_{C}$ following the procedure described in Section 3.2.

The test mixtures in this study were prepared in a pre-vacuumed stainless-steel mixing tank at room temperature by filling the tank with fuel, $\mathrm{O}_{2}$, and $\mathrm{N}_{2}$ one at a time. As iC8 and iC12 are liquids at room 
temperature, a glass syringe is used to inject the fuel into the mixing tank first. Then $\mathrm{O}_{2}$ and $\mathrm{N}_{2}$ are filled into the mixing tank consecutively based on barometric measurements. After filling the reactants, the heaters and magnetic stirrer are switched on, 2-4 hours are needed to reach the desired initial temperature $\left(T_{0}=373-413 \mathrm{~K}\right.$ in this study) and are allowed to completely vaporize the liquid fuel as well as to let the entire system reach steady state. Vaporization check conducted for iC12 demonstrates that more than $95 \%$ of $\mathrm{iC} 12$ is vaporized in the mixing tank within two minutes, even at a lower $T_{0}$ of $368 \mathrm{~K}$, as shown in Fig. $\mathrm{S} 1$ in Supplementary Material. The molar ratio of $\mathrm{N}_{2}$ and $\mathrm{O}_{2}$ is kept at 3.76 throughout this study. The molar proportions of the mixtures and the corresponding test conditions of iC8 and iC12 experiments investigated are summarized in Table 1. Ignition delay time measurements for iC8/air and iC12/air mixtures are conducted at compressed pressures of $P_{C}=15,20$, and $30 \mathrm{bar}$, and at equivalence ratios of $\phi=0.7,1.0$, and 2.0. The test matrix is designed to provide experimental data over a wider equivalence ratio and pressure range for chemical kinetic model development and validation. High purity iC8 $(99.8 \%), \mathrm{iC} 12(>99 \%)$, and $\mathrm{O}_{2} / \mathrm{N}_{2}(>99.99 \%)$ used to prepare the test mixtures are from Sigma-Aldrich, Haltermann Solutions, and Airgas, respectively.

\section{Computational Specifications}

\subsection{Chemical Kinetic Model of iC8 and iC12}

The detailed chemical kinetic model of $\mathrm{iC} 8$ and $\mathrm{iC} 12$ is constructed based on the recent high temperature iso-alkanes modeling work of Guzman et al. [42] with newly added low temperature kinetics of isoalkanes. As some reactions involving the fuel radicals of iC12, e.g., reactions with iso-butene, lead to the iC16 pathways, the oxidation chemistry of iC16 is also considered herein. Briefly, the current model is built hierarchically on the model of Zhang et al. [43] for alkanes and the $\mathrm{C}_{0}-\mathrm{C}_{4}$ base chemistry of AramcoMech2.0 [44]. This model describes both the high temperature and low temperature kinetics of iC8 and iC12 using a consistent set of reaction pathways and the associated reaction rates. The reaction rates for the high temperature reaction classes 1-9, as described by Curran et al. [31], have largely been 
taken from the earlier work of Mehl et al. [33], with an exception to $\mathrm{H}$-abstraction via $\dot{O} H$ which was taken from the work of Badra et al. [45]. The rates for the low temperature reaction classes 10-25, as described by Curran et al. [31], which are the low temperature chain branching peroxy reactions, have been adopted from the ab-initio works of [46-49]. In addition to the above-mentioned 25 classes, the reaction classes corresponding to the concerted $\mathrm{HO}_{2}$ elimination of alkylperoxy radicals (commonly referred as ROÓ radicals) and of hydroperoxyl alkylhydroperoxides (commonly referred as ȮOQOOH radicals), as well as the alternative isomerization reactions of OOQOOH radicals producing the dihydroperoxy alkyl radicals (commonly referred as $\dot{\mathrm{P}}(\mathrm{OOH})_{2}$ radicals), have also been modeled. Those additional low temperature reaction pathways are for revising the details of the chain branching, chain propagation, and chain termination functions in the model. It is necessary to point out that the reaction pathways of $\dot{\mathrm{P}}(\mathrm{OOH})_{2}$ are largely based on analogies from $\dot{\mathrm{Q} O O H}$. As the isomerization reaction of $\dot{\mathrm{O} O Q O O H} \Leftrightarrow \dot{\mathrm{P}}(\mathrm{OOH})_{2}$ is similar to $\dot{\mathrm{R}} \mathrm{O}_{2} \Leftrightarrow \dot{\mathrm{QOOH}}$, analogies from the latter are adopted to describe the formation of $\dot{\mathrm{P}}(\mathrm{OOH})_{2}$. Likewise, as $\dot{\mathrm{P}}(\mathrm{OOH})_{2}$ and $\dot{\mathrm{Q} O O H}$ radicals are similar in nature, the $\dot{\mathrm{P}}(\mathrm{OOH})_{2}$ radicals have been modeled to undergo reactions identical to those of $\dot{\mathrm{OOOH}}$ radicals and analogies are used for describing the reactions of $\dot{\mathrm{P}}(\mathrm{OOH})_{2}$ radicals. Furthermore, the decomposition of KHP and HPCE is one of the important reaction classes in the low temperature chemistry, but the rate for this reaction of large KHPs and HPCEs (larger than $\mathrm{C}_{5}$ ) unfortunately has not been studied theoretically, and the rate constant expressions used for this reaction class in the literature show significant variations with pre-Arrhenius (A) factor varying from $5 \times 10^{15}$ to $3 \times 10^{16}\left(\mathrm{~s}^{-1}\right)$, and activation energies $\left(\mathrm{E}_{\mathrm{a}}\right)$ between 39 and $43 \mathrm{kcal} / \mathrm{mole}[4,8,25,31-33,36,43,44,50,51]$. For the current mechanism, we used $A=10^{16} \mathrm{~s}^{-1}$ and $E_{a}=41.2 \mathrm{kcal} /$ mole, which are close to the mean of the rate expressions used in the literature mechanisms. The reaction schematic of iso-alkanes that the current model of iC8 and iC12 follows is shown in Fig. 3, in which the important low temperature chain branching reactions are marked in green and the major inhibiting reactions are marked in red. The sources of important low temperature 
reaction classes are also summarized in Table 2. In addition, the optimizations of the reaction rate rules used in this study are summarized in Table S1 in the Supplementary Material.

Furthermore, the thermodynamic properties of the intermediate species have been estimated using the group additivity method with the recent group values taken from Burke et al. [52]. As the iso-alkanes studied herein are severely branched, the Gauche interactions and H-1,5 interactions are also counted during the estimation of the thermodynamic properties. The procedure used for counting those interactions is similar to that used in the study of Atef et al. [25].

\subsection{Analytical methods}

Two types of simulations are performed using the Python interface of Cantera 2.3.0 [41] in this study. The first type is 'RCM simulation' that accounts for the machine-specific effect by modeling it as volume change [40]. The detailed descriptions of the RCM simulations can be found in Weber and Sung [38] and Dames et al. [53]. Briefly, a combined pressure trace is used to deduce the time history of reactor volume. The combined pressure trace uses the pressure profile during the compression stroke (before the EOC) from the reactive run, while the pressure profile after the EOC is taken from the corresponding non-reactive experiment. This procedure ensures that the combined pressure trace faithfully represents the reactor conditions during the compression stroke, while enabling modeling of the reactor conditions after the EOC. Cantera [41] is used to convert the combined pressure trace to a volume trace by assuming that the mixture undergoes an isentropic compression during the compression stroke and an isentropic expansion after the EOC. The resulting volume trace is then fed to the IdealGasReactor in Cantera [41] to generate a simulated reactive pressure trace, from which the $\tau_{1}$ and $\tau$ values of RCM simulation are obtained following the same procedure of determining experimental ignition delay times described in Section 2. Furthermore, by turning off the reactions (i.e., setting the reaction multiplier to zero) during RCM simulation, the temperature at the EOC is recorded as $T_{C}$.

The second type of simulation is 'CONV simulation' that uses a constant-volume, adiabatic reactor, which is the IdealGasReactor in Cantera [41] without accounting for changes in the reactor volume. The 
simulated $\tau\left(\tau_{1}\right)$ values are defined as the time required for the simulated temperature to increase by 400 $\mathrm{K}(10 \mathrm{~K})$ over the initial temperature in the simulation. It is found that the ignition delay time based on the temperature increase is close to that determined using the time derivative of pressure. The CONV simulations in this study are mainly used to perform ST simulations and model-based analyses. The nonideal facility-dependent effects of ST simulation [54,55] are not considered in this work.

Besides ignition delay time simulations, model-based analyses are also carried out, such as reaction pathway analyses and sensitivity analyses. Reaction pathway analyses are based on constant volume simulation using Cantera [41], with the major species and their possible reaction pathways determined based on the rate of destruction (ROD) and rate of production (ROP) of each species through each reaction at a selected time index. The branching percent of a target reaction from a certain species is defined as the ratio of the ROD of this species in a target reaction divided by the total destruction rate of this species, which is calculated as follows:

$$
\text { Branching Percent }(\%)=\frac{R O D_{t}}{\sum_{i}^{N} R O D_{i}} \times 100 \%,
$$

where $N$ is the number of reactions in the model, $R O D_{t}$ is the ROD through the target reaction, and $R O D_{i}$ is the ROD through the $i$-th reaction.

Sensitivity analyses of ignition delay times to the reaction rate coefficient of each reaction are performed using constant volume simulations in Cantera [41]. By perturbing the pre-exponential factor of the target reaction by a factor of 2 , the corresponding $\tau^{+}$is obtained with the perturbed rate coefficient of $k^{+}$. The sensitivity coefficient for $\tau$ with the unperturbed reaction rate coefficient of $k$ is then defined as: $S_{\tau}=\ln \left(\tau^{+} / \tau\right) / \ln \left(k^{+} / k\right)$. In addition, the sensitivity analyses of $\tau_{1}$ are conducted in the same manner. As such, the reaction with a positive (negative) value of sensitivity coefficient retards (promotes) the overall reactivity. 


\section{Results and Discussion}

\subsection{Comparison with Literature Data and Model}

Figure 4 compares the experimental ignition delay times of stoichiometric iC8/air and iC12/air mixtures at 20 bar obtained from the current RCM experiments and the literature RCM data of iC8 [25] and iC12 [36], as well as the ST data for iC8 [14] and iC12 [34,36]. Overall, the current RCM measurements agree/complement well with the literature RCM/ST data. Regarding the RCM data comparison for iC8, while small discrepancies in the low-temperature regime (LTR) and the negative temperature coefficient (NTC) regime are noticed between the previous and current measurements, the newly-acquired $\tau$ and $\tau_{1}$ of iC8 generally match well with those reported in [25], especially considering varying heat transfer characteristics and initial temperature/pressure conditions in different RCM experiments. With respect to the RCM data comparison for iC12, the current total ignition delay data agree well with those reported in [36]. As the first-stage ignition delay data were not reported in [36], the current datasets of $\tau_{1}$ provide the much needed information for validating the low-temperature chemistry of iC12.

Using the chemical kinetic model developed in this study, the available RCM and ST data are modeled using 'RCM simulation' and 'CONV simulation', respectively. Figure 4 shows that the simulated results agree well with the experimental $\tau$ and $\tau_{1}$ of $\mathrm{iC} 8$ and $\mathrm{iC} 12$, illustrating the predictive capability of the current model over a wide range of temperatures. It has to be pointed out in both iC8 and $\mathrm{iC} 12$ cases that 'CONV simulation' over-predicts the literature ST data by about $30 \%$ for iC8 and $50 \%$ for iC12. For the ST data with $\tau$ beyond $1 \mathrm{~ms}$, the main discrepancy source is likely the non-ideal, facility-dependent effects in the ST experiments that cause pressure increases of 2-4\%/ms [14]. Including the facility-dependent effects in the ST simulations are expected to improve the agreement between simulated and experimental results. At higher temperatures when the ST-measured $\tau$ becomes less than $1 \mathrm{~ms}$, the reactivity of the oxidation of large fuels is controlled by the oxidation of the $\mathrm{C}_{1}-\mathrm{C}_{4}$ fragments, which is the AramcoMech2.0 [44] in the current model. Further improvements of the $\mathrm{C}_{1}-\mathrm{C}_{4}$ chemistry are expected to reduce the discrepancy level at high temperatures. 
In addition, the simulated results using the iC12 kinetic model developed by Mao et al. [36] are included in Fig. 4(b) for comparison. As shown in Fig. 4(b), the model of [36] displays noticeable discrepancies compared to the RCM data while the current model demonstrates better performance for both $\tau$ and $\tau_{1}$ prediction. Specifically, the model of [36] agrees well with the experimental $\tau$ at low temperatures but under-predicts $\tau$ in the NTC regime and intermediate temperature regime (ITR). It is also noted that although the 'CONV simulation' results using the model of [36] appear to agree well with the literature ST data in Fig. 4(b), considering the non-ideal facility-dependent effects in the ST experiments as discussed previously, the model of [36] likely over-predicts the iC12 reactivity at high temperatures. Moreover, the model of [36] over-predicts the experimental $\tau_{1}$ up to a factor of 3 , while the current model performs better. This further indicates that the model of [36] under-predicts the second-stage ignition delay times at low temperatures.

\subsection{Experimental and Simulated Ignition Delay Times}

Figure 5 shows the measured $\tau_{1}$ and $\tau$ for iC8/air and iC12/air mixtures investigated herein. For both iC 8 and iC12 at varying test conditions, $\tau$ decreases with increasing $T_{C}$ in the LTR and ITR, while it increases with increasing $T_{C}$ in the NTC regime. On the other hand, $\tau_{1}$ decreases monotonically with increasing $T_{C}$. The magnitude of the NTC response, reflected by its slope in the Arrhenius plot, is also different between iC8 and iC12. At the same conditions of $P_{C}$ and $\phi, \mathrm{iC} 8$ is seen to have a steeper slope in Fig. 5, and hence the NTC response of iC8 is stronger than iC12. In addition, Figs. 5(a) and 5(c) demonstrate the effect of pressure on ignition delay times of stoichiometric iC8 and iC12 mixtures in air. In general, both $\tau_{1}$ and $\tau$ decrease with increasing $P_{C}$, with $\tau$ showing stronger pressure sensitivity than $\tau_{1}$. It is further noted that the NTC response becomes weaker when increasing $P_{C}$ to 30 bar for both iC 8 and iC12. At $P_{C}=20$ bar, Figs. 5(b) and 5(d) show the effect of equivalence ratio on ignition delay times for iC8 and iC12. Since "air" is used as the oxidizer here, the effect of equivalence ratio represents the effect of fuel loading. In general, for both iC8 and iC12, increasing $\phi$ (i.e. fuel concentration) decreases $\tau$ for a given $T_{C}$, while $\tau_{1}$ is far less sensitive to the change in $\phi$ as compared to $\tau$. 
Using the current chemical kinetic model, RCM simulations of $\mathrm{iC} 8$ and $\mathrm{iC} 12$ are also conducted at varying conditions. Overall, the simulated results of $\tau_{1}$ and $\tau$ show good agreement with experimental data at varying $P_{C}$ and $\phi$ for both iC8 and iC12. Nonetheless, some discrepancies are still noticed at certain test conditions. For iC8 in the ITR, the current model under-predicts $\tau$ at $P_{C}=15$ and 20 bar but over-predicts $\tau$ at $P_{C}=30$ bar, as shown in Fig. 5(a). For iC12, the simulated $\tau_{1}$ and $\tau$ in the LTR are slightly longer than the RCM data, especially at $P_{C}=30$ bar as shown in Fig. 5(c). These observations suggest that the pressure dependence of some low-to-intermediate temperature reactions in the present model may require further refinements. Despite the above-mentioned discrepancies, the performance of the current model is reasonable, illustrating its predictive capability as well as its potential of being used in understanding the autoignition characteristics of $\mathrm{iC} 8$ and $\mathrm{iC} 12$.

Figure 6 further compares the current RCM data of iC8/air and iC12/air mixtures at varying test conditions in order to provide an insight into the differences and similarities between the autoignition of iC8 and iC12 at low-to-intermediate temperatures. It is seen from Fig. 6 that the $\tau$ of iC8 is consistently longer than that of $\mathrm{iC} 12$ in the LTR/ITR; however, in the NTC regime the $\tau$ of iC8 becomes shorter than that of iC12. This indicates that there is a reactivity crossover between iC8 and iC12 at different temperature regimes. As shown in Fig. 6 , iC12 has consistently shorter $\tau_{1}$ than $\mathrm{iC} 8$, which causes the $\tau$ of $\mathrm{iC} 12$ to be shorter than that of $\mathrm{iC} 8$ at low temperature $(<650 \mathrm{~K})$. In contrast, the second-stage ignition delay times of iC12 are longer than those of iC8 at low temperatures $(<720 \mathrm{~K})$, and the difference increases as temperature increases. As a result, the $\tau$ of iC12 gradually exceeds that of iC 8 as temperature increases, thereby leading to the first reactivity crossover. As discussed earlier, iC8 exhibits a stronger NTC response than $\mathrm{iC} 12$, and hence $\tau$ of $\mathrm{iC} 8$ increases more rapidly than $\mathrm{iC} 12$ does as temperature increases in the NTC regime, which eventually leads to the second reactivity crossover near the end of the NTC. It is worth noting that the experiments of iC8 and iC12 in this study are performed using the same RCM, and their heat transfer characteristics are similar at the same test condition $\left(\phi, T_{C}\right.$, $\left.P_{C}\right)$. Thus, the observed reactivity crossover is not affected by different heat transfer effects between the 
$\mathrm{RCM}$ experiments of $\mathrm{iC} 8$ and $\mathrm{iC} 12$. As such, it is of fundamental importance to carry out further chemical kinetic analyses in order to explicate the following experimental observations accounting for reactivity crossover - $\mathrm{iC} 12$ has a shorter $\tau_{1}$, iC12 has longer second-stage ignition delay times below $720 \mathrm{~K}$, and iC8 has a stronger NTC response.

Comparing the simulated $\tau$ between iC8 and iC12 in Fig. 6, the experimentally observed reactivity crossover is successfully captured by the current model at varying test conditions. This further substantiates the potential of the current model as a tool to understand the autoignition of iC8 and iC12 at low temperatures. It is of interest to note that the above-mentioned reactivity crossover between iC 8 and $\mathrm{iC} 12$ is not observed when comparing the simulated ignition delay times of $n$-octane (nC8) and $n$ dodecane (nC12) reported in $[3,4]$, in which the simulated $\tau$ results of $n$-alkanes are shown to slightly decrease with increasing carbon number over a wide range of temperatures. While $n$-alkanes share the similar molecular structure as carbon number increases, the molecular structures of iso-alkanes can vary vastly as carbon number increases, which may lead to different low temperature pathways. Thus, the reactivity crossover between $\mathrm{iC} 8$ and $\mathrm{iC} 12$ is possibly caused by their own unique molecular structures.

Figure 7 further demonstrates the experimental and simulated pressure trace comparisons for stoichiometric iC8/air and iC12/air mixtures at $P_{C}=15$ bar with three representative temperatures of $T_{C} \sim$ $645 \mathrm{~K}, 710 \mathrm{~K}$, and $837 \mathrm{~K}$, representing the LTR, NTC regime, and ITR, respectively. It can be observed from Fig. 7(a) that both iC8 and iC12 exhibit two-stage ignition behavior in the LTR. In the NTC regime for $T_{C^{\sim}} 710 \mathrm{~K}$, iC8 exhibits a two-stage ignition behavior with very short $\tau_{1}$, while iC12 displays singlestage ignition behavior. Further increasing $T_{C}$ to $837 \mathrm{~K}$ (ITR), both iC8 and iC12 exhibit single-stage ignition behavior. It can also be clearly observed that there is a reactivity crossover between iC8 and iC12 as temperature increases from LTR to ITR, as shown in Fig. 6(a). Namely, iC8 has shorter $\tau$ than iC12 in the NTC regime, but becomes longer in the LTR and ITR. It is also noted that the machine settings in the RCM experiments for $\mathrm{iC} 8$ and $\mathrm{iC} 12$ shown in Fig. 7(a) are similar. As the values of heat of combustion on a unit mass of fuel basis are similar for iC8 and iC12, their peak pressures after hot 
ignition are similar ( $\sim 64$ bar). Furthermore, it has to be pointed out that the observed differences in the post-hot-ignition peak pressure values between iC8 and iC12 in Figs. 7(b) and 7(c) are caused by the different machine settings used in the respective RCM experiments in order to achieve the desired compressed conditions for each fuel. Comparing the modeled first-stage and hot-ignition pressure rises with experiments, Fig. 7 shows the model well captured the first-stage pressure rise. The hot-ignition pressure rise, on the other hand, is over-predicted by the model for both iC8 and iC12 at all $T_{C}$, especially at $837 \mathrm{~K}$. Those overpredictions can be attributed to the non-ideality of the experimental facility (not truly zero-dimensional). The heat and mass transfer from the adiabatic core region to the crevice during hot-ignition is expected to reduce the hot-ignition pressure rise in experiments. This effect becomes even stronger under the RCM machine settings with smaller reactor volumes at EOC, which typically happens in the high $T_{C}$ cases.

\subsection{Reaction Pathway and Brute-Force Sensitivity Analyses}

To provide an overview of the current model, reaction pathway and brute-force sensitivity analyses are conducted and shown in Figs. 8 and 9, respectively. As the reaction pathways of iC8 are better known, Fig. 8 only shows those of iC12. The reaction pathway analyses of iC12 have been performed at $10 \%$ fuel consumption prior to any ignition activity, with $\phi=1.0$ in air, initial pressure of 20 bar, and initial temperatures of $650 \mathrm{~K}, 750 \mathrm{~K}$, and $850 \mathrm{~K}$ respectively representing LTR, NTC, and ITR. The reaction pathway analyses of iC8 can be found in Fig. S2 in Supplementary Material. It is noted that reactions with branching percent less than 5\% at all three initial temperatures are not shown in Fig. 8 and Fig. S2. In addition, the molecular structures of the important species are shown in Fig. 8 and Fig. S2.

Since both iC8 and iC12 share the same skeleton as shown in Fig. 3, their reaction pathways display similar patterns. The main low temperature pathways start with the fuel $\mathrm{H}$-abstraction reactions $\left(r_{l}\right)$, with both $\mathrm{iC} 8$ and iC12 forming four different fuel radicals $(\dot{\mathrm{R}})$. Subsequently, $\dot{\mathrm{R}}$ radicals undergo the first $\mathrm{O}_{2}$ addition process $\left(r_{2}\right)$ to form alkyl-peroxyl radicals (ROO), which are then isomerizes to produce 
Q $\mathrm{OOH}\left(r_{3}\right)$. The following second $\mathrm{O}_{2}$ addition process $\left(r_{4}\right)$ allows the formation of OOQOOH, which enables the consecutive chain branching steps: the formation of KHP $\left(r_{5}\right)$ and the decomposition of KHP $\left(r_{6}\right)$, producing two $\dot{\mathrm{OH}}$ in total. In parallel with the KHP channel, the HPCE chain branching channel starts from $\dot{\mathrm{OOQOOH}}$ isomerization $\left(r_{7}\right)$ that produces $\dot{\mathrm{P}}(\mathrm{OOH})_{2}$ radicals. The formed $\dot{\mathrm{P}}(\mathrm{OOH})_{2}$ radicals then enable the consecutive chain branching steps: the formation of HPCE $\left(r_{8}\right)$ and the decomposition of HPCE $\left(r_{9}\right)$, producing two $\dot{\mathrm{OH}}$ in total. These reactions can affect the low temperature reactivity drastically because of the net $\dot{\mathrm{OH}}$ production resulting from the chain branching steps. Hence, the main low temperature pathways of $\mathrm{iC} 8 / \mathrm{iC} 12$ are expected to have promoting effects on both $\tau$ and $\tau_{1}$. Note that each type of $\dot{O} O Q O O H$ radicals can form serval types of $\dot{P}(\mathrm{OOH})_{2}$ radicals $\left(r_{7}\right)$ with different radical site locations. Moreover, each $\dot{\mathrm{P}}(\mathrm{OOH})_{2}$ radical may also decompose to different HPCEs $\left(r_{8}\right)$ via different transition states. Those features lead to 38 possible $\dot{\mathrm{P}}(\mathrm{OOH})_{2}$ radical structures and 37 possible structures of HPCEs in the current model. To compare the HPCE channel and the KHP channel side by side, the branching percent values of the $\dot{\mathrm{P}}(\mathrm{OOH})_{2}$ formation and the HPCE formation shown in Fig. 8 are the combined branching percent values of their related $\dot{\mathrm{P}}(\mathrm{OOH})_{2}$ radicals and HPCEs.

There are several types of side reactions in Fig. 8 that compete with the main low temperature pathways. The concerted elimination reactions $\left(r_{13}\right)$ compete with the ROO isomerization pathways for ROÖ and produce alkenes which are relatively "stable" at temperatures in the LTR and NTC regime. Similarly, concerted elimination reactions $\left(r_{14}\right)$ compete with the KHP and HPCE channels for OOOOOH and produce "stable" alkenes. The formation reactions of cyclic ethers $\left(r_{11}\right)$ compete with the second $\mathrm{O}_{2}$ addition to $\dot{\mathrm{Q} O O H}$ reactions. At high temperatures, the $\beta$-scission reactions $\left(r_{10}\right)$ become more important and break down fuel-radicals directly without going through the low-temperature pathways, and hence reduce the $\dot{\mathrm{OH}}$ production rate from the low temperature pathways.

The importance of the above-mentioned reactions in both iC 8 and iC12 is also reflected in the brute force sensitivity analyses, performed under the same conditions as the reaction pathway analyses, as 
shown in Fig. 9. The key reactions are selected based on the magnitude of their sensitivity coefficients. The top-ranked 15 reactions on each side (promoting or inhibiting) at $750 \mathrm{~K}$, plus a few reactions only ranked top at $650 \mathrm{~K}$ or $850 \mathrm{~K}$ are presented in Fig. 9. It is seen that the fuel $\mathrm{H}$-abstraction reactions $\left(r_{l}\right)$ are generally among the highest impact reactions in both promoting and inhibiting the reactivity of iC8 and iC12 in the LTR and NTC regime. Specifically, the H-abstraction reactions $\left(r_{1}\right)$ that form fuelradicals with primary and secondary carbons tend to have promoting effects while the fuel-radicals with tertiary carbon tend to strongly inhibit the reactivity. This is because the fuel-radicals with tertiary carbons are unable to enter the KHP chain branching channel and thus have much less contribution to $\dot{\mathrm{OH}}$ production. Moreover, the concerted elimination reactions $\left(r_{13}\right)$ can only take place on the site of tertiary carbon, as shown in Fig. 8, which also have strong inhibiting effects as shown in Fig. 9. Therefore, the tertiary carbon channel presents the strongest inhibiting effect on reactivity for both iC8 and $\mathrm{iC} 12$. As the KHP and HPCE channels provide the essential ÖH source, the reactions leading to the KHP and HPCE channels also exhibit strong promoting effects, including the first and second $\mathrm{O}_{2}$ addition $\left(r_{2}, r_{4}\right)$, ROO isomerization $\left(r_{3}\right)$, and dissociations of KHP $\left(r_{6}\right)$ and HPCE $\left(r_{9}\right)$. The strong inhibiting reactions include the production reactions of cyclic ethers $\left(r_{11}\right)$ because the resulting net $\dot{\mathrm{OH}}$ production is decreased, as discussed previously. In the ITR, represented by $850 \mathrm{~K}$, a different sequence of reactions becomes important in the sensitivity analyses. This sequence of $\mathrm{H}$-atom abstraction from iC8 and iC12 by $\mathrm{HO}_{2}$ radicals forms hydrogen peroxide $\left(\mathrm{H}_{2} \mathrm{O}_{2}\right)$ which decomposes to two reactive $\dot{\mathrm{O}} \mathrm{H}$ radicals, thereby greatly promoting reactivity.

The sensitivity analysis results of Fig. 9 also reveal the distinct effects of some competing pathways for iC8 and iC12. For instance, the isomerization reaction of ROO radicals $\left(r_{3}\right)$ such as $\mathrm{XC12H} 25 \mathrm{O} 2-$ $3 \Leftrightarrow \mathrm{XC12OOH} 3-1$ shows strong promoting effect because it is competing with the concerted elimination reaction $\mathrm{XC12} \mathrm{H} 25 \mathrm{O} 2-3 \Leftrightarrow \mathrm{HO} 2+\mathrm{XC12H} 24-3$. Similarly, the second $\mathrm{O}_{2}$ addition reactions also present promoting effect due to their competition with the formation of cyclic ethers, which can be 
found in the sensitivity analysis results of both iC8 and iC12. In addition, the Q $O O H$ isomerization reaction $\mathrm{XC12H} 12 \mathrm{O} 2-1 \Leftrightarrow \mathrm{XC12OOH} 1-3$ is found to inhibit the reactivity which is different from the majority of other $\mathrm{Q} O O H$ isomerization reactions. This is due to its subsequent cyclic ether production

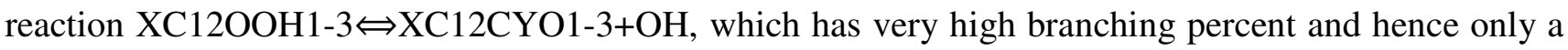
small amount of XC12OOH1-3 can enter the later low-temperature chain branching pathways. As such,

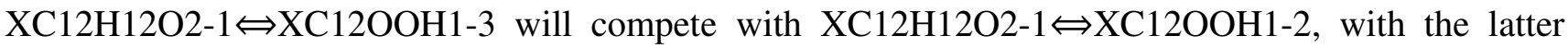
path having a larger percent going into the low-temperature chain branching pathways. The result of this competition is the inhibiting effect of $\mathrm{XC12H} 12 \mathrm{O} 2-1 \Leftrightarrow \mathrm{XC12OOH} 1-3$, and the counterpart reaction in $\mathrm{iC} 8, \mathrm{IC} 8-1 \mathrm{O} 2 \mathrm{R} \Leftrightarrow \mathrm{IC} 8 \mathrm{OOH} 1-4 \mathrm{R}$, also shows similar strong inhibiting effect.

As temperature increases, the dominating reaction pathways have changed for both iC8 and iC12. As shown in Fig. 8, the branching percent values of the first $\mathrm{O}_{2}$ addition reactions $\left(r_{2}\right)$ are reduced as temperature increases while the branching percent values of the $\beta$-scission reactions $\left(r_{10}\right)$ show the opposite trend. This can be attributed to two reasons: higher temperatures lower the stability of ROO adducts and overcome the energy barriers of the $\beta$-scission reactions. Those combined effects cause the branching percent changes as temperature increases. The cyclic ether production reactions $\left(r_{11}\right)$ are also favored at higher temperatures, as reflected by their branching percent values in Fig. 8. This is due to the competing second $\mathrm{O}_{2}$ addition reactions $\left(r_{4}\right)$ also have reduced rates as temperature increases, similar to the first $\mathrm{O}_{2}$ addition reactions. In addition, the concerted elimination reactions $\left(r_{13}\right)$ have higher branching percent values at higher temperatures because the increase in temperature helps to overcome the energy barriers. Furthermore, the sensitivity coefficients of the KHP and HPCE dissociation reactions in Fig. 9, such as $\mathrm{XC} 12 \mathrm{KET} 2-2 \mathrm{P} \Rightarrow \mathrm{CH} 3 \mathrm{CHCO}+\mathrm{OH}+\mathrm{TC} 4 \mathrm{H} 9+\mathrm{TC} 4 \mathrm{H} 9 \mathrm{CHO}$, reduce drastically as temperature increases, indicating that those reactions only govern the low temperature reactivity. The details regarding the KHP pathways will be discussed in due course. 


\subsection{First-Stage Ignition Reactivity Comparison}

To understand the differences in $\tau_{1}$ between iC 8 and iC12, the underlying first-stage ignition chemistry is first analyzed. Figure 10 demonstrates the sensitivity analysis results of $\tau_{1}$ at initial conditions of 20 bar and $650 \mathrm{~K}$. Based on the magnitude of the $\tau_{1}$ sensitivity coefficients, the top ranked 10 reactions on each side (positive or negative sensitivity coefficient) are presented in Fig. 10. For both iC8 and iC12, it is seen that the decompositions of KHP and HPCE play an important role in promoting $\tau_{1}$ because of their ability to provide low temperature chain branching pathways. As the decomposition reactions of KHP and HPCE have relatively high activation energies, their rates are much lower than the rates of their corresponding formation reactions at low temperatures, indicating that KHP and HPCE will encounter an accumulation process before the first-stage ignition.

The sequence of the first-stage ignition event can be explained as follows. As fuel oxidation proceeds, KHP (and HPCE) is produced without much decomposition at low temperatures. With increasing KHP (HPCE) concentration and the mild temperature rise during the fuel oxidation process, the decomposition rate of KHP (HPCE) gradually increases. As a result, KHP (HPCE) will first accumulate to a certain limit and beyond which the decomposition rate of KHP (HPCE) exceeds its production rate. Since the decomposition of KHP (HPCE) produces $\dot{\mathrm{O} H}$, the overall reactivity is promoted. The resulting heat release then further accelerates the KHP (HPCE) decomposition in a positive feedback loop. Consequently, a burst of $\dot{\mathrm{OH}}$ production is created right after the KHP (HPCE) net ROP peaks, thereby triggering the first-stage ignition. As the first-stage ignition progresses and the accumulated KHP (HPCE) is consumed, the $\dot{\mathrm{OH}}$ production rate as well as the heat release rate drop, signifying the end of first-stage ignition. Meanwhile, the first-stage heat release elevates the system temperature such that the decomposition of KHP (HPCE) is fast enough and it ceases to accumulate. Therefore, the decomposition of the accumulated KHP (HPCE) triggers the first-stage ignition event and how fast the KHP (HPCE) accumulation can reach its concentration limit determines the $\tau_{1}$ for both iC8 
and iC12. Even with the similar first-stage ignition chemistry, iC12 is shown to have shorter $\tau_{1}$ than $\mathrm{iC} 8$; the reasons for that are discussed in the following.

Figure 11 shows and compares the net rate of production (ROP) of KHP, including that of the most dominant KHP species and the total for all KHP isomers, for stoichiometric iC8 and iC12 in air at initial conditions of 20 bar and $650 \mathrm{~K}$. It can be observed that iC12 reaches the peak net ROP of KHP sooner than iC8 does, which can be attributed to the fuel molecular structure effect. As shown in Fig. 12, iC8 has only one secondary carbon while $\mathrm{iC} 12$ has two. Hence, the secondary-secondary (ss) carbon pathway that $\mathrm{OOQOOH}$ goes through to form KHP in the iC12 chemistry is a channel that cannot be found in the iC8 chemistry. As an example, Fig. 12 also shows the fastest KHP producing pathways for iC8 and iC12, which are the IC8KET5-3 pathway and the XC12KET2-2P pathway, respectively. This KHP producing pathway for iC12 starts from $\mathrm{XC12OOH2-2PO2} \mathrm{(ODOOOH)} \mathrm{and} \mathrm{goes} \mathrm{through} \mathrm{the}$ secondary-secondary (ss) carbon pathway. More specifically, a 6-member transition state is first formed between two secondary carbons and enables the subsequent $\mathrm{H}$-abstraction on the left-hand-side of the secondary carbon site. The product then quickly undergoes $\beta$-scission to release XC12KET2-2P (KHP) and one $\dot{\mathrm{O}} \mathrm{H}$. The similar process for $\mathrm{iC} 8$, however, must go through the secondary-primary (sp) carbon pathway, which has a higher activation energy when forming the 6-member transition state. As such, the entire process of $\mathrm{iC} 12$ from $\mathrm{XC12OOH} 2-2 \mathrm{PO} 2(\mathrm{O} O \mathrm{OOOH})$ to $\mathrm{XC12KET2-2P}$ (KHP) is roughly 10 times faster than its $\mathrm{iC} 8$ counterpart. It is also worth to note that the process from OOQOOH to KHP is expressed as a single step reaction in the current model. The corresponding rate constant comparison among the major KHP production pathways for both $\mathrm{iC} 8$ and $\mathrm{iC} 12$ can be found in Fig. S3 in Supplementary Material, which demonstrates that the XC12KET2-2P production pathway for iC12 is much faster than the IC8KET5-3 production pathway for iC8. In view of the above, an explanation for iC12 to have shorter $\tau_{1}$ is because it has a faster KHP production rate than iC 8 does. 
As an alternative pathway to the KHP channel, the HPCE channel also plays a similar role in the low temperature chemistry of both iC 8 and iC 12 , which tends to increase the reactivity of both iC 8 and iC12. This increase in reactivity is a consistent finding from other $C_{5}-C_{7}$ alkane mechanisms $[25,50,51]$. To further illustrate the impact of the HPCE pathway on the current iC8-iC12 model, the formation pathways $\left(r_{7}\right)$ of its precursor $\dot{\mathrm{P}}(\mathrm{OOH})_{2}$ for both $\mathrm{iC} 8$ and $\mathrm{iC} 12$ are removed from the original model. Then, the CONV simulated $\tau_{1}$ and $\tau$ are compared to those obtained using the original model, as shown in Fig. S6 in Supplementary Material. It is found the overall reactivity of both iC8 and iC12 at temperatures below $900 \mathrm{~K}$ is noticeably reduced by removing the $\dot{\mathrm{P}}(\mathrm{OOH})_{2}$ formation pathways. The additional reactivity introduced by the $\dot{\mathrm{P}}(\mathrm{OOH})_{2}$ pathways is deemed significant for better agreement against experimental $\tau$ and $\tau_{1}$ for both $\mathrm{iC} 8$ and $\mathrm{iC} 12$.

The HPCE pathways exhibit other interesting trends. The ROPs of iC8 and iC12 do not show a clear structural dependency as the KHP channel demonstrates in Figs. 11 and 12. In addition, iC8 and iC12 have similar $\tau_{1}$ dependence on the inclusion of the HPCE pathways (cf. Fig. S6), indicating the HPCE pathways have a negligible contribution to the $\tau_{1}$ difference observed between iC8 and iC12 (cf. Fig. 6). While the chemical kinetic effects of the HPCE channel are not investigated in-depth herein, its significance in iso-alkane oxidation cannot be overlooked and merits further studies.

\subsection{NTC Chemistry Comparison}

In the current model, the NTC behaviors of $\mathrm{iC} 8$ and $\mathrm{iC} 12$ are dominated by two types of reactions: the cyclic ether pathways $\left(r_{11}, r_{12}\right)$ and the concerted elimination pathways $\left(r_{13}, r_{14}\right)$. To provide an insight into the reactivity crossover between $\mathrm{iC} 8$ and $\mathrm{iC} 12$ in the NTC regime, the role of the cyclic ether pathways in the NTC behaviors of $\mathrm{iC} 8$ and $\mathrm{iC} 12$ is of particular interest and analyzed in the following.

As discussed previously, the inhibiting effect of cyclic ethers on autoignition is caused by their consumption of $\dot{\mathrm{QOOH}}\left(r_{11}\right)$ which competes with the second $\mathrm{O}_{2}$ addition pathways $\left(r_{4}\right)$. The second $\mathrm{O}_{2}$ addition pathways $\left(r_{4}\right)$ have strong promoting effects because they enable the later low temperature 
chain-branching steps $\left(r_{5}, r_{6}, r_{8}, r_{9}\right)$ and net $\dot{\mathrm{O}} \mathrm{H}$ production. The production of cyclic ethers mainly occurs during the first-stage ignition for the cases with two-stage ignition response or prior to hot ignition for the single-stage ignition cases. As the vast destruction of cyclic ethers only occurs near hot ignition, the cyclic ethers produced during the induction period have no contribution to the growth of radical pool, thereby leading to strong inhibiting effect. Moreover, the sensitivity analyses for both iC8 and $\mathrm{iC} 12$ show that the major cyclic ether production pathways have higher positive values of sensitivity for $\tau$ compared to $\tau_{1}$; see Fig. S4 in Supplementary Material. As such, the cyclic ether pathways mainly influence the second-stage ignition delay time, which is essential in understanding the reactivity crossover between $\mathrm{iC} 8$ and $\mathrm{iC} 12$.

Figure 13 demonstrates the branching percent of $\dot{Q} 00 H$ entering the cyclic ether pathway and the second $\mathrm{O}_{2}$ addition pathway at the time of $10 \%$ fuel consumption as a function of initial temperature for stoichiometric iC8 and iC12 in air under initial pressure of 20 bar. The choice of $10 \%$ fuel consumption is because this corresponds to the start of first-stage ignition in the presence of two-stage ignition response so that the cyclic ether production can be better represented. It is seen that the branching percent of the second $\mathrm{O}_{2}$ addition pathway $\left(r_{4}\right)$ decreases with increasing temperature as discussed earlier, indicating its reduced promoting effect as temperature increases. In addition, the branching percent of the cyclic ether pathway $\left(r_{11}\right)$ increases with increasing temperature, indicating its stronger inhibiting effect at higher temperatures. As a result, the combined effect leads to a reduced reactivity of both iC8 and iC12 with increasing temperature. Further comparing iC8 and iC12 in Fig. 13, the branching percent of the cyclic ether pathway in $\mathrm{iC} 12$ is consistently higher than that in iC8 below 800 $\mathrm{K}$, indicating a stronger inhibiting effect from the cyclic ether pathway in $\mathrm{iC} 12$ that results in a longer second-stage ignition delay time. This could be one possible reason why iC12 shows a longer $\tau$ than iC8 in the temperature range of $650-750 \mathrm{~K}$. 
Moreover, Fig. 13 shows that iC8 exhibits a stronger temperature dependence of the branching percent (reflected by the steeper slope) for both the cyclic ether pathway and the second $\mathrm{O}_{2}$ addition pathway than $\mathrm{iC} 12$. This indicates that the reduction in branching percent with increasing temperature is more drastic for iC8 compared to iC12. This could be a reason why iC8 has a stronger NTC response than $\mathrm{iC} 12$ does, which results in a longer $\tau$ for iC8 beyond $750 \mathrm{~K}$. As temperature increases above $900 \mathrm{~K}$, high temperature chemistry takes over and the above-mentioned molecular structure effects are no longer important. Hence, the $\tau$ difference between iC 8 and iC 12 diminishes beyond $900 \mathrm{~K}$.

Among cyclic ethers, oxiranes (3-member rings), oxetanes (4-member rings), and tetrahydrofurans (5-member rings) can be formed through the cyclic ether pathways in iC8 and iC12, with THFs (short for tetrahydrofurans) being the most favored cyclic ether due to its less ring strain that results in faster production rate [45]. To investigate the dominating cyclic ether production pathways for both iC8 and iC12, net ROP analyses of cyclic ethers have been conducted at $\phi=1.0$ in air under initial conditions of 20 bar and $750 \mathrm{~K}$, as shown in Fig. 14. It can be observed that the dominant cyclic ethers produced in iC8 and iC12 are iC8O1-4 and XC12CYO1-3, respectively, with both being substituted-THFs as expected. Figure S5 in Supplementary Material further shows the rate constants of the four fastest cyclic ether production reactions for both $\mathrm{iC} 8$ and $\mathrm{iC} 12$. The cyclic ether production reaction with the largest rate constant also have the highest net ROP value. Specifically, the rate constant of the reaction producing $\mathrm{XC12CYO1-3}$ from $\mathrm{XC12OOH1-3}$ is about 10 times larger than that of the reaction producing IC8O1-4 from IC8OOH1-4R, and it is even larger than the rate constants of the rest of cyclic ether production reactions.

The 'Other paths' denoted in Fig. 13 include the $\beta$-scission reactions of $\beta$-Q்OH $\left(r_{15}\right)$ and the QOOH unimolecular decomposition $\left(r_{16}\right)$ which compete with the cyclic ether pathway $\left(r_{11}\right)$ and the second $\mathrm{O}_{2}$ addition pathway $\left(r_{4}\right)$ for $\mathrm{Q} O \mathrm{OH}$. However, for both $\mathrm{iC} 8$ and $\mathrm{iC} 12$ over the temperature range investigated, their branching percent values of 'Other paths' are seen to overlap and may not have a 
strong influence on the reactivity crossover between $\mathrm{iC} 8$ and iC12. In addition to the reactions described in Fig.13, the concerted elimination reactions $\left(r_{13}, r_{14}\right)$ also contribute to the NTC behaviors greatly for both iC8 and iC12. However, unlike the cyclic ether pathway which affects $\tau$ more than $\tau_{1}$ (i.e. it mainly affects the second-stage ignition delay time), the concerted elimination reactions $\left(r_{13}, r_{14}\right)$ show a similar influence on $\tau_{1}$ and $\tau$ in both iC8 and iC12, as shown in the sensitivity analysis results of Fig. S4 in Supplementary Material. This indicates that the concerted elimination reactions $\left(r_{13}, r_{14}\right)$ may not have large influences on the reactivity crossover between $\mathrm{iC} 8$ and $\mathrm{iC} 12$ as the cyclic ether pathway. Moreover, no molecular structure dependency has been observed from the concerted elimination reactions $\left(r_{13}, r_{14}\right)$ for both $\mathrm{iC} 8$ and $\mathrm{iC} 12$. As a result, the influence of the concerted elimination reactions on the NTC behaviors of $\mathrm{iC} 8$ and $\mathrm{iC} 12$ is not further analyzed in this work.

\section{Conclusions}

The autoignition experiments of iso-octane (iC8) and iso-dodecane (iC12) in air are performed in this study using a rapid compression machine at varying compressed pressures and equivalence ratios. Both iC8 and iC12 show a similar autoignition trend that the total ignition delay time decreases with increasing temperature in the low temperature regime (LTR) and the intermediate temperature regime (ITR) but increases as temperature increases in the negative temperature coefficient (NTC) regime. These two iso-alkanes also demonstrate similar responses to the pressure and equivalence ratio variations: the total ignition delay time decreases with increasing pressure and equivalence ratio. On the other hand, the first-stage ignition delay times are less sensitive than the total ignition delay time to the variations of pressure and equivalence ratio.

Comparing the newly-acquired $\mathrm{iC} 8$ and iC12 data, they all exhibit NTC response but in different extent, with iC8 showing stronger NTC response. The total ignition delay times of iC12 are shorter than iC8 in the ITR. In the LTR, both the total ignition delay times and first-stage ignition delay times of iC8 are longer than iC12. Furthermore, the total ignition delay time crossover between iC8 and iC12 is observed in the NTC regime over the conditions investigated. This, in turn, suggests that there are more 
complicated chemical kinetics involved in the NTC regime for these two iso-alkanes due to their different molecular structures that lead to the observed reactivity crossover.

In addition, a detailed chemical kinetic model of iC8 and iC12 has been developed in this study and validated against the newly-acquired autoignition data. Overall, the current model presents reasonable agreement with experimental data at varying test conditions with small discrepancies likely caused by the missing pressure dependence for some reactions. Moreover, the reactivity crossover in the NTC regime between iC8 and iC12 and the general first-stage ignition delay trends have been successfully captured by the current model. Model-based analyses show that the reason causing first-stage ignition is mainly due to the competition of the accumulation and decomposition of ketohydroperoxides (KHP) and hydroperoxy cyclic ethers (HPCE). Moreover, in the LTR one possible reason for iC8 to have longer first-stage and total ignition delay times than $\mathrm{iC} 12$ is due to the molecular structure differences between iC8 and iC12 such that the fastest KHP (HPCE) pathway of iC12 is $~ 10$ times faster than that of iC8. Model-based analysis also indicates that the reactivity crossover between iC 8 and iC12 is related to their differences in the branching percent of $\dot{Q} O O H$ consumption through the cyclic ether pathways and the net rate of production of the reactions producing tetrahydrofurans.

This study provides a validation database for model development and insights into the fuel molecular structure effects on autoignition characteristics of two selected highly-branched iso-alkanes (iC8 and iC12) at low-to-intermediate temperatures. Future experimental and modeling investigations involving more different branched-chain alkanes are required to achieve comprehensive understanding of low temperature combustion of highly branched hydrocarbons. 


\section{Acknowledgements}

The work at UCONN was supported by Lawrence Livermore National Laboratory via Standard Research Subcontract No. B630575 and the National Science Foundation under Grant No. CBET1402231. The work at LLNL was performed under the auspices of the U.S. Department of Energy (DOE), Contract DE-AC52-07NA27344, and was supported by the U.S. Department of Energy, Vehicle Technologies Office (Program Managers: Mike Weismiller and Gurpreet Singh).

\section{Supplementary Material}

Supplementary Material consists of (1) Figures S1-S6 and Table S1, (2) species dictionary of important species for iC8 and iC12 at low temperatures, (3) the chemical kinetic model developed in this work, in both Chemkin and Cantera formats, (4) a summary of the current ignition delay time data, and (5) RCM experimental pressure traces and the deduced time-volume histories for RCM simulations, which are available from the website of the Combustion Diagnostics Laboratory at UCONN (https://combdiaglab.engr.uconn.edu/database/rcm-database). 


\section{References}

[1] W.J. Pitz, C.J. Mueller, Recent progress in the development of diesel surrogate fuels, Prog. Energy Combust. Sci. 37 (2011) 330-350.

[2] S. Dooley, S.H. Won, et al., A jet fuel surrogate formulated by real fuel properties, Combust. Flame 157 (2010) 2333-2339.

[3] C.K. Westbrook, W.J. Pitz, O. Herbinet, H.J. Curran, E.J. Silke, A comprehensive detailed chemical kinetic reaction mechanism for combustion of n-alkane hydrocarbons from n-octane to nhexadecane, Combust. Flame 156 (2009) 181-199.

[4] S.M. Sarathy, C.K. Westbrook, et al., Comprehensive chemical kinetic modeling of the oxidation of 2-methylalkanes from C7 to C20, Combust. Flame 158 (2011) 2338-2357.

[5] S. Dooley, S.H. Won, J. Heyne, T.I. Farouk, Y. Ju, F.L. Dryer, K. Kumar, X. Hui, C.J. Sung, H. Wang, M.A. Oehlschlaeger, V. Iyer, S.S. Iyer, T. Litzinger, R.J. Santoro, T. Malewicki, K. Brezinsky, The experimental evaluation of a methodology for surrogate fuel formulation to emulate gas phase combustion kinetic phenomena, Combust. Flame 159 (2012) 1444-1466.

[6] F. Battin-Leclerc, Detailed chemical kinetic models for the low-temperature combustion of hydrocarbons with application to gasoline and diesel fuel surrogates, Prog. Energy Combust. Sci. 34 (2008) 440-498.

[7] A.K. Agarwal, A.P. Singh, R.K. Maurya, Evolution, challenges and path forward for low temperature combustion engines, Prog. Energy Combust. Sci. 61 (2017) 1-56.

[8] F. Zhao, T.N. Asmus, D.N. Assanis, J.E. Dec, J.A. Eng, P.M. Najt, Homogeneous Charge Compression Ignition (HCCI) Engines, SAE International, United States, 2003.

[9] K. Fieweger, R. Blumenthal, G. Adomeit, Shock-tube investigations on the self-ignition of hydrocarbon-air mixtures at high pressures, Symp. (Int.) Combust. 25 (1994) 1579-1585.

[10] K. Fieweger, R. Blumenthal, G. Adomeit, Self-ignition of S.I. engine model fuels: a shock tube investigation at high pressure, Combust. Flame 109 (1997) 599-619.

[11] D.F. Davidson, M.A. Oehlschlaeger, J.T. Herbon, R.K. Hanson, Shock tube measurements of isooctane ignition times and $\mathrm{OH}$ concentration time histories, Proc. Combust. Inst. 29 (2002) 12951301.

[12] M.A. Oehlschlaeger, D.F. Davidson, J.T. Herbon, R.K. Hanson, Shock tube measurements of branched alkane ignition times and $\mathrm{OH}$ concentration time histories, Int. J. Chem. Kinet. 36 (2004) 67-78.

[13] D.F. Davidson, B.M. Gauthier, R.K. Hanson, Shock tube ignition measurements of iso-octane/air and toluene/air at high pressures, Proc. Combust. Inst. 30 (2005) 1175-1182. 
[14] H.-P.S. Shen, J. Vanderover, M.A. Oehlschlaeger, A shock tube study of iso-octane ignition at elevated pressures: the influence of diluent gases, Combust. Flame 155 (2008) 739-755.

[15] M. Hartmann, I. Gushterova, M. Fikri, C. Schulz, R. Schieß1, U. Maas, Auto-ignition of toluenedoped $n$-heptane and iso-octane/air mixtures: high-pressure shock-tube experiments and kinetics modeling, Combust. Flame 158 (2011) 172-178.

[16] T. Malewicki, A. Comandini, K. Brezinsky, Experimental and modeling study on the pyrolysis and oxidation of iso-octane, Proc. Combust. Inst. 34 (2013) 353-360.

[17] J.F. Griffiths, P.A. Halford-Maw, D.J. Rose, Fundamental features of hydrocarbon autoignition in a rapid compression machine, Combust. Flame 95 (1993) 291-306.

[18] A. Cox, J.F. Griffiths, C. Mohamed, H.J. Curran, W.J. Pitz, C.K. Westbrook, Extents of alkane combustion during rapid compression leading to single-and two-stage ignition, Symp. (Int.) Combust. 26 (1996) 2685-2692.

[19] C.V. Callahan, T.J. Held, F.L. Dryer, R. Minetti, M. Ribaucour, L.R. Sochet, T. Faravelli, P. Gaffuri, E. Rani, Experimental data and kinetic modeling of primary reference fuel mixtures, Symp. (Int.) Combust. 26 (1996) 739-746.

[20] R. Minetti, M. Carlier, M. Ribaucour, E. Therssen, L.R. Sochet, Comparison of oxidation and autoignition of the two primary reference fuels by rapid compression, Symp. (Int.) Combust. 26 (1996) 747-753.

[21] X. He, M.T. Donovan, B.T. Zigler, T.R. Palmer, S.M. Walton, M.S. Wooldridge, A. Atreya, An experimental and modeling study of iso-octane ignition delay times under homogeneous charge compression ignition conditions, Combust. Flame 142 (2005) 266-275.

[22] X. He, B.T. Zigler, S.M. Walton, M.S. Wooldridge, A. Atreya, A rapid compression facility study of $\mathrm{OH}$ time histories during iso-octane ignition, Combust. Flame 145 (2006) 552-570.

[23] S.M. Walton, He X., B.T. Zigler, M.S. Wooldridge, A. Atreya, An experimental investigation of iso-octane ignition phenomena, Combust. Flame 150 (2007) 246-262.

[24] G. Mittal, C.J. Sung, A rapid compression machine for chemical kinetics studies at elevated pressures and temperatures, Combust. Sci. Technol. 179 (2007) 497-530.

[25] N. Atef, G. Kukkadapu, S.Y. Mohamed, M.A. Rashidi, C. Banyon, M. Mehl, K.A. Heufer, E.F. Nasir, A. Alfazazi, A.K. Das, C.K. Westbrook, W.J. Pitz, T. Lu, A. Farooq, C.J. Sung, H.J. Curran, S.M. Sarathy, A Comprehensive iso-octane combustion model with improved thermochemistry and chemical kinetics, Combust. Flame 178 (2017) 111-134.

[26] G.M. Côme, V. Warth, P.A. Glaude, R. Fournet, F. Battin-LeClerc, G. Scacchi, Computer-aided design of gas-phase oxidation mechanisms-Application to the modeling of n-heptane and isooctane oxidation, Proc. Combust. Inst. 26 (1996) 755-762. 
[27] C.E. Roberts, R.D. Matthews, W.R. Leppard, Development of a semi-detailed kinetics mechanism for the autoignition of iso-octane", Diagnostics and Modeling in SI Engines, Publ. SAE-962107, Society of Automotive Engineers (1996).

[28] S.G. Davis, C.K. Law, Laminar flame speeds and oxidation kinetics of iso-octane-air and $n$ heptane-air flames, Proc. Combust. Inst. 27 (1998) 521-527.

[29] H.J. Curran, W.J. Pitz, C.K. Westbrook, C. Callahan, F.L. Dryer, Oxidation of automotive primary reference fuels at elevated pressures, Proc. Combust. Inst. 27 (1998) 379-387.

[30] J.-S. Chen, T.A. Litzinger, H.J. Curran, The lean oxidation of iso-octane in the intermediate temperature regime at elevated pressures, Combust. Sci. and Technol. 155 (2000) 49-79.

[31] H.J. Curran, P. Gaffuri, W.J. Pitz, C.K. Westbrook, A comprehensive modeling study of iso-octane oxidation, Combust. Flame 129 (2002) 253-280.

[32] M. Mehl, W.J. Pitz, M. Sjöberg, J.E. Dec, Detailed kinetic modeling of low-temperature heat release for PRF fuels in an HCCI engine, Report No. 2009-01-1806, SAE International, Warrendale, PA, 2009.

[33] M. Mehl, W.J. Pitz, C.K. Westbrook, H.J. Curran, Kinetic modeling of gasoline surrogate components and mixtures under engine conditions, Proc. Combust. Inst. 33 (2011) 193-200.

[34] S.H. Won, F.M. Haas, A. Tekawade, G. Kosiba, M.A. Oehlschlaeger, S. Dooley, F.L. Dryer, Combustion characteristics of $\mathrm{C} 4$ iso-alkane oligomers: Experimental characterization of isododecane as a jet fuel surrogate component. Combust. Flame 165 (2016) 137-143.

[35] G. Flora, J. Balagurunathan et al., Chemical ignition delay of candidate drop-in replacement jet fuels under fuel-lean conditions: A shock tube study. Fuel 209 (2017) 457-472.

[36] Y. Mao, Y. Feng, Z. Wu, S. Wang, L. Yu, M. Raza, Y. Qian, X. Lu, The autoignition of isododecane in low to high temperature range: An experimental and modeling study. Combust. Flame 210 (2019) 222-235.

[37] A.K. Das, C.J. Sung, Y. Zhang, G. Mittal, Ignition delay study of moist hydrogen/oxidizer mixtures using a rapid compression machine, Int. J. Hydrogen Energy 37 (2012) 6901-6911.

[38] B.W. Weber, C.J. Sung, UConnRCMPy: Python-Based Data Analysis for Rapid Compression Machines, $10^{\text {th }}$ U.S. National Combustion Meeting (2017), paper 2D19.

[39] D. Lee, S. Hochgreb, Rapid Compression Machines: Heat transfer and suppression of corner vortex, Combust. Flame 114 (1998) 531-545.

[40] C.J. Sung, H.J. Curran, Using rapid compression machines for chemical kinetics studies, Prog. Energy Combust. Sci. 44 (2014) 1-18.

[41] D.G. Goodwin, H.K. Moffat, R.L. Speth, Cantera: An object-oriented software toolkit for chemical kinetics, thermodynamics, and transport processes. Version 2.3.0 (2017). 
[42] J. Guzman, G. Kukkadapu, K. Brezinsky, C. Westbrook, Experimental and modeling study of the pyrolysis and oxidation of an iso-paraffinic alcohol-to-jet fuel, Combust. Flame 201 (2019) 57-64.

[43] K. Zhang, C. Banyon, U. Burke, G. Kukkadapu, S.W. Wagnon, M. Mehl, H.J. Curran, C.K. Westbrook, W.J. Pitz, An experimental and kinetic modeling study of the oxidation of hexane isomers: developing consistent reaction rate rules for alkanes, Combust. Flame 206 (2019) 123137.

[44] Y. Li, C.W. Zhou, K.P. Somers, K. Zhang, and H.J. Curran, The oxidation of 2-butene: A high pressure ignition delay, kinetic modeling study and reactivity comparison with isobutene and 1butene, Proc. Combust. Inst. 36 (2017) 403-411.

[45] J. Badra, A. Farooq, Site-specific reaction rate constant measurements for various secondary and tertiary H-abstraction by OH radicals, Combust. Flame 162 (2015) 2034-2044.

[46] A. Miyoshi, Systematic computational study on the unimolecular reactions of alkylperoxy $\left(\mathrm{RO}_{2}\right)$, hydroperoxyalkyl $(\mathrm{QOOH})$, and hydroperoxyalkylperoxy $(\mathrm{O} 2 \mathrm{QOOH})$ radicals, J. Phys. Chem. A 115 (2011) 3301-3325.

[47] S.M. Villano, L.K. Huynh, H.H. Carstensen, and A.M. Dean, High-pressure rate rules for alkyl + $\mathrm{O} 2$ reactions. 1. The dissociation, concerted elimination, and isomerization channels of the alkyl peroxy radical, J. Phys. Chem. A 115 (2011) 13425-13442.

[48] S.M. Villano, L.K. Huynh, H.H. Carstensen, and A.M. Dean, High-pressure rate rules for alkyl + $\mathrm{O}_{2}$ reactions. 2. The isomerization, cyclic ether formation, and $\beta$-scission reactions of hydroperoxy alkyl radicals, J. Phys. Chem. A 116 (2012) 5068-5089.

[49] S. Sharma, S. Raman, W.H. Green, Intramolecular hydrogen migration in alkylperoxy and hydroperoxyalkylperoxy radicals: Accurate treatment of hindered rotors, J. Phys. Chem. A 114 (2010) 5689-5701.

[50] J. Bugler, B. Marks, O. Mathieu, R. Archuleta, A. Camou, C. Grégoire, K.A. Heufer, E.L. Petersen, H.J. Curran, An ignition delay time and chemical kinetic modeling study of the pentane isomers, Combust. Flame 163 (2016) 138-156.

[51] S.Y. Mohamed, L. Cai, F. Khaled, C. Banyon, Z. Wang, M.J. Al Rashidi, H. Pitsch, H.J. Curran, A. Farooq, S.M. Sarathy, Modeling ignition of a heptane isomer: improved thermodynamics, reaction pathways, kinetics, and rate rule optimizations for 2-methylhexane, J. Phys. Chem. A 120 (2016) 2201-2217.

[52] S.M. Burke, J.M. Simmie, H.J. Curran, Critical evaluation of thermochemical properties of $\mathrm{C}_{1}-\mathrm{C}_{4}$ species: Updated group-contributions to estimate thermochemical properties, J. Physical and Chemical Reference Data 44 (2015) 013101. 
[53] E.E. Dames, A.S. Rosen, B.W. Weber, C.W. Gao, C.J. Sung, W.H. Green, A detailed combined experimental and theoretical study on dimethyl ether/propane blended oxidation, Combust. Flame 168 (2016) 310-330.

[54] D.F. Davidson, R.K. Hanson, Recent advances in shock tube/laser diagnostic methods for improved chemical kinetics measurements, Shock Waves 19 (2009) 271-283.

[55] G.A. Pang, D.F. Davidson, R.K. Hanson, Experimental study and modeling of shock tube ignition delay times for hydrogen-oxygen-argon mixtures at low temperatures, Proc. Combust. Inst. 32 (2009) 181-188. 


\section{Figures:}

$$
i \mathrm{C} 8
$$

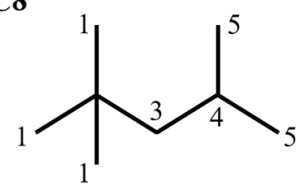

2,2,4-trimethylpentane (iso-octane) $i \mathrm{C} 12$

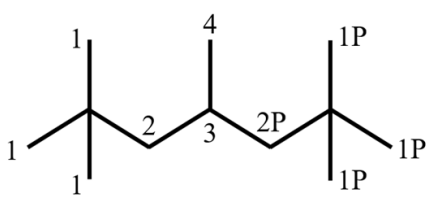

2,2,4,6,6-pentamethylheptane (iso-dodecane)

Figure 1: Molecule structures of iso-octane (iC8) and iso-dodecane (iC12). Carbon labels are used later in defining species names.
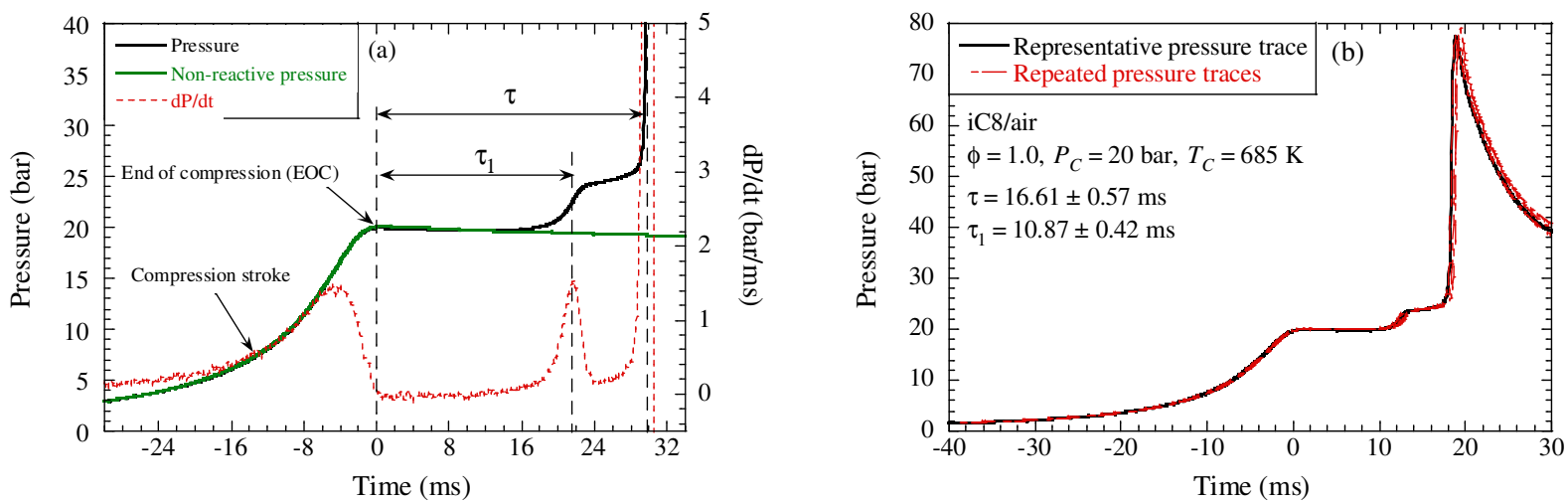

Figure 2: (a) Experimental pressure traces demonstrating the definitions of $\tau_{1}$ and $\tau$ with stoichiometric iC12/air at $P_{C}=20$ bar and $T_{C}=647 \mathrm{~K}$. The corresponding non-reactive pressure trace by replacing $\mathrm{O}_{2}$ with $\mathrm{N}_{2}$ is also shown as a reference. (b) Representative and repeated experimental pressure traces with stoichiometric iC8/air at $P_{C}=20$ bar and $T_{C}=685 \mathrm{~K}$. 


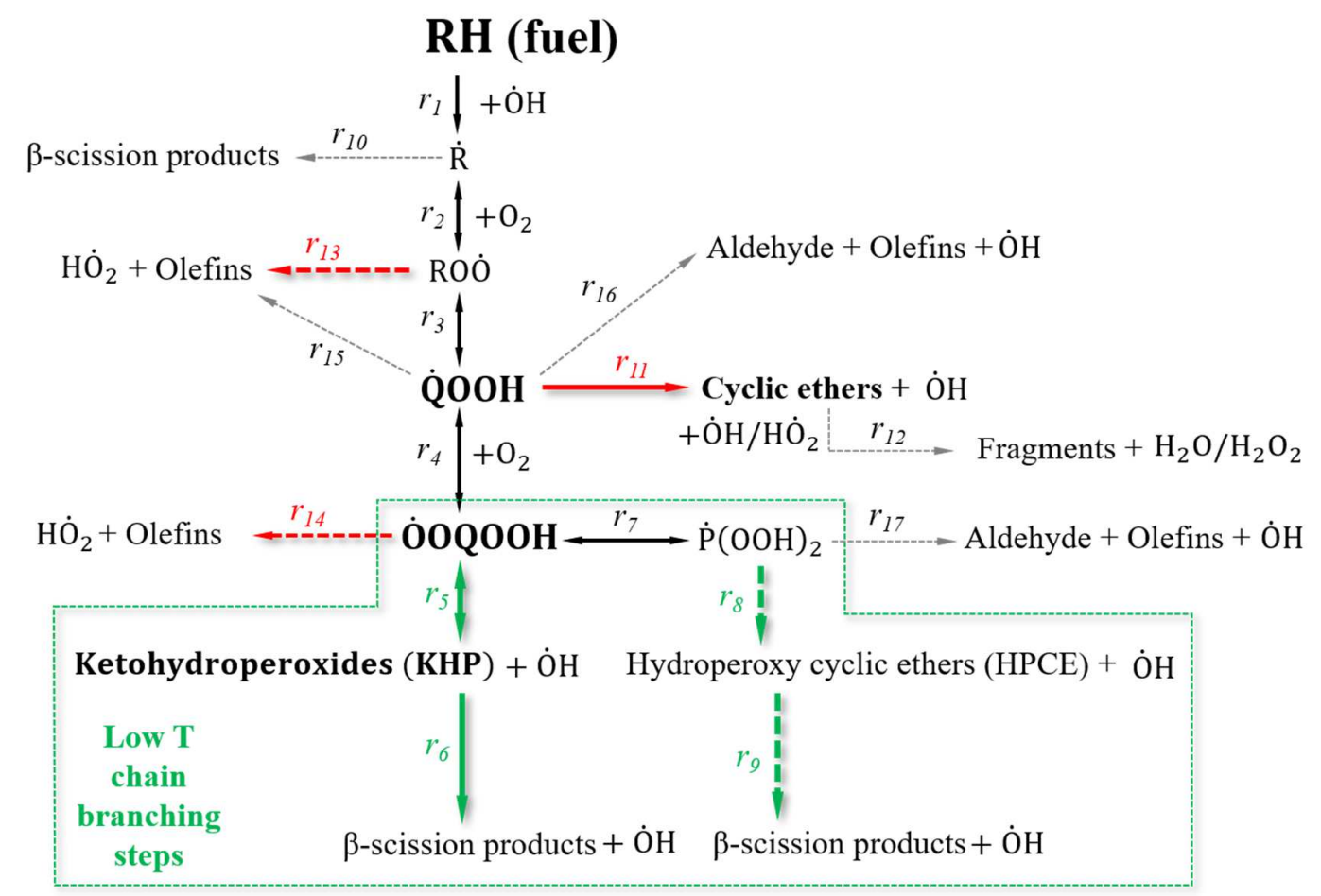

Figure 3: The iso-alkanes reaction schematic followed by the current iC8 and iC12 model. The index $r_{i}$ denotes the $i$ th reaction class. Green (Red) arrows indicate dominating promoting (inhibiting) reaction classes at low-to-intermediate temperatures.
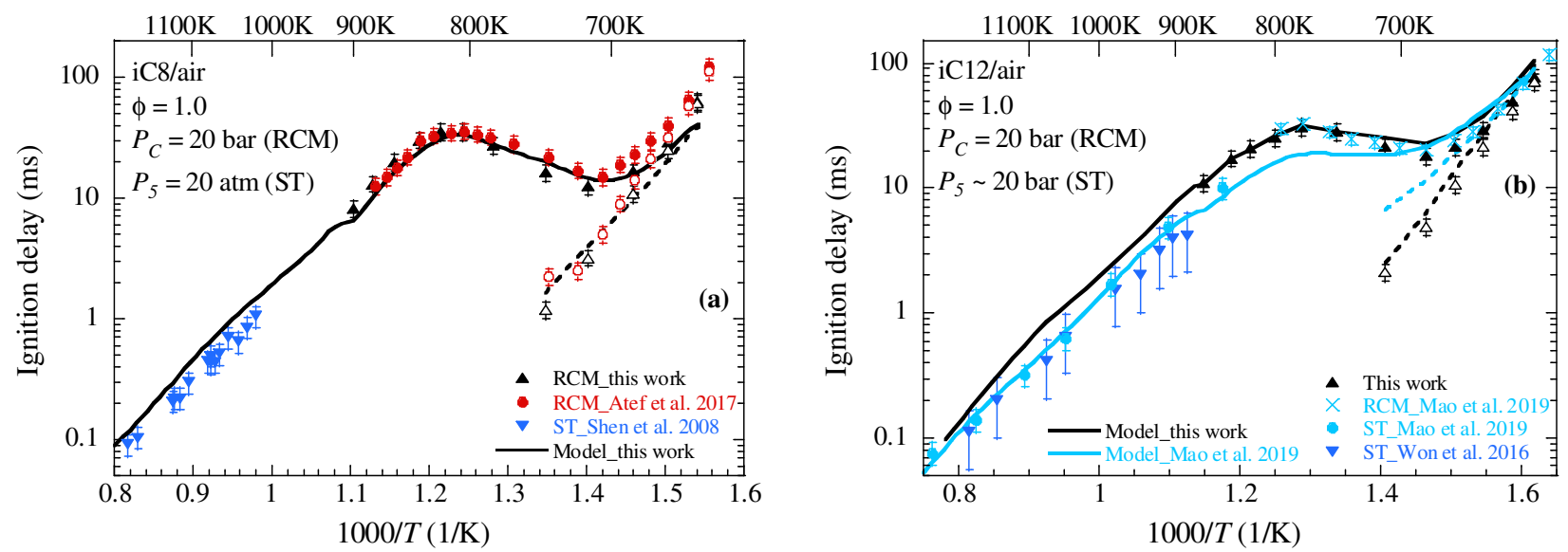

Figure 4: Comparison of ignition delay times of stoichiometric (a) iC8/air and (b) iC12/air mixtures between the current RCM study and the literature RCM and ST data. Experimental results are shown as symbols. Filled symbols: total ignition delay times. Open symbols: first-stage ignition delay times. Simulated results are presented as lines. Solid lines: total ignition delay times. Dashed lines: first-stage ignition delay times. 

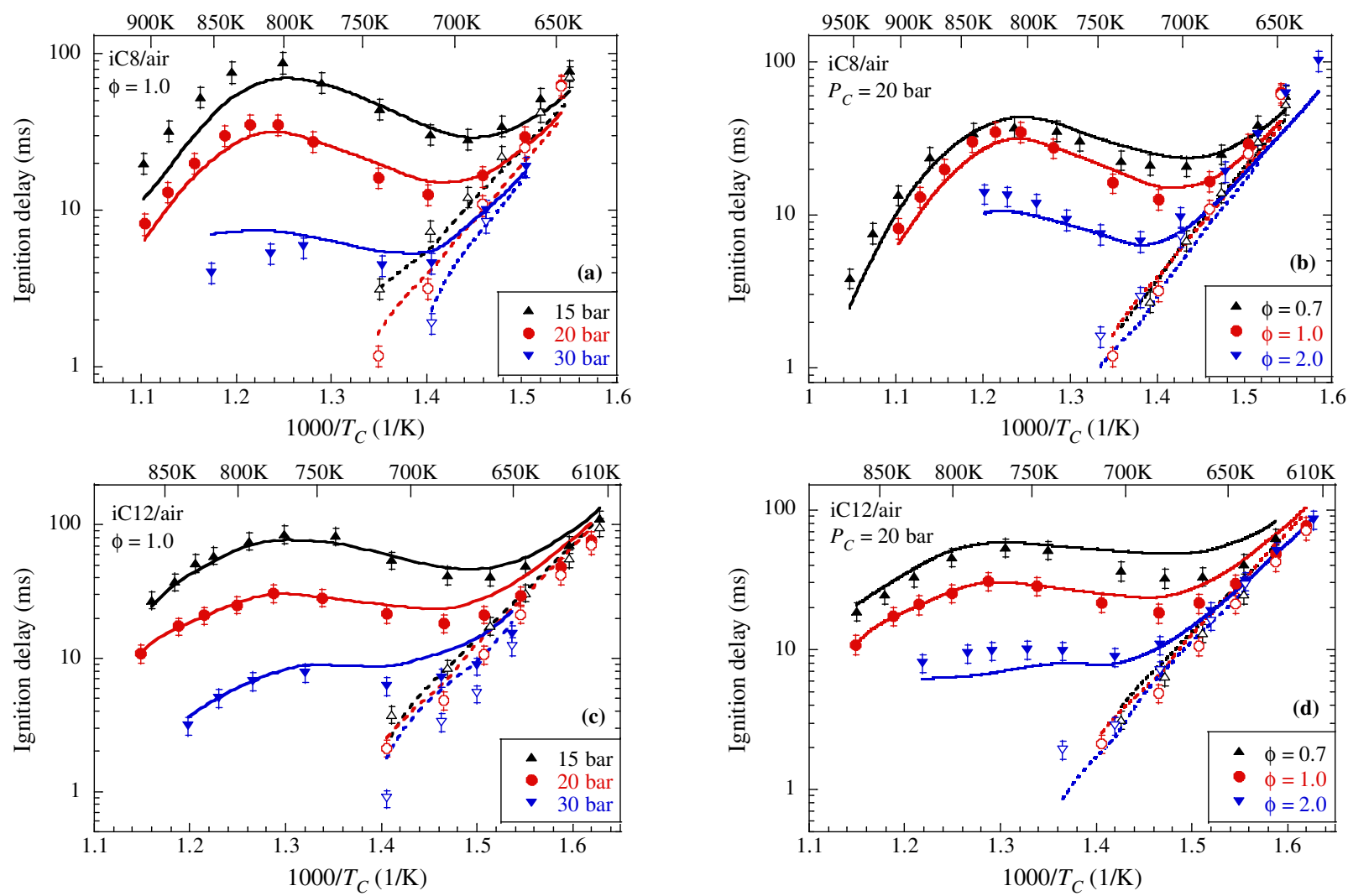

Figure 5: Experimental and simulated ignition delay times of $\mathrm{iC} 8$ and $\mathrm{iC} 12$ at varying $\phi$ and $P_{C}$. (a) iC8, $\phi=1.0$, and varying $P_{C}$; (b) iC8, $P_{C}=20$ bar, and varying $\phi$; (c) iC12, $\phi=1.0$, and varying $P_{C}$; (d) iC12, $P_{C}=20$ bar, and varying $\phi$. Experimental results are shown as symbols. Filled symbols: total ignition delay times. Open symbols: first-stage ignition delay times. Simulated results are presented as lines. Solid lines: total ignition delay times. Dashed lines: first-stage ignition delay times. 

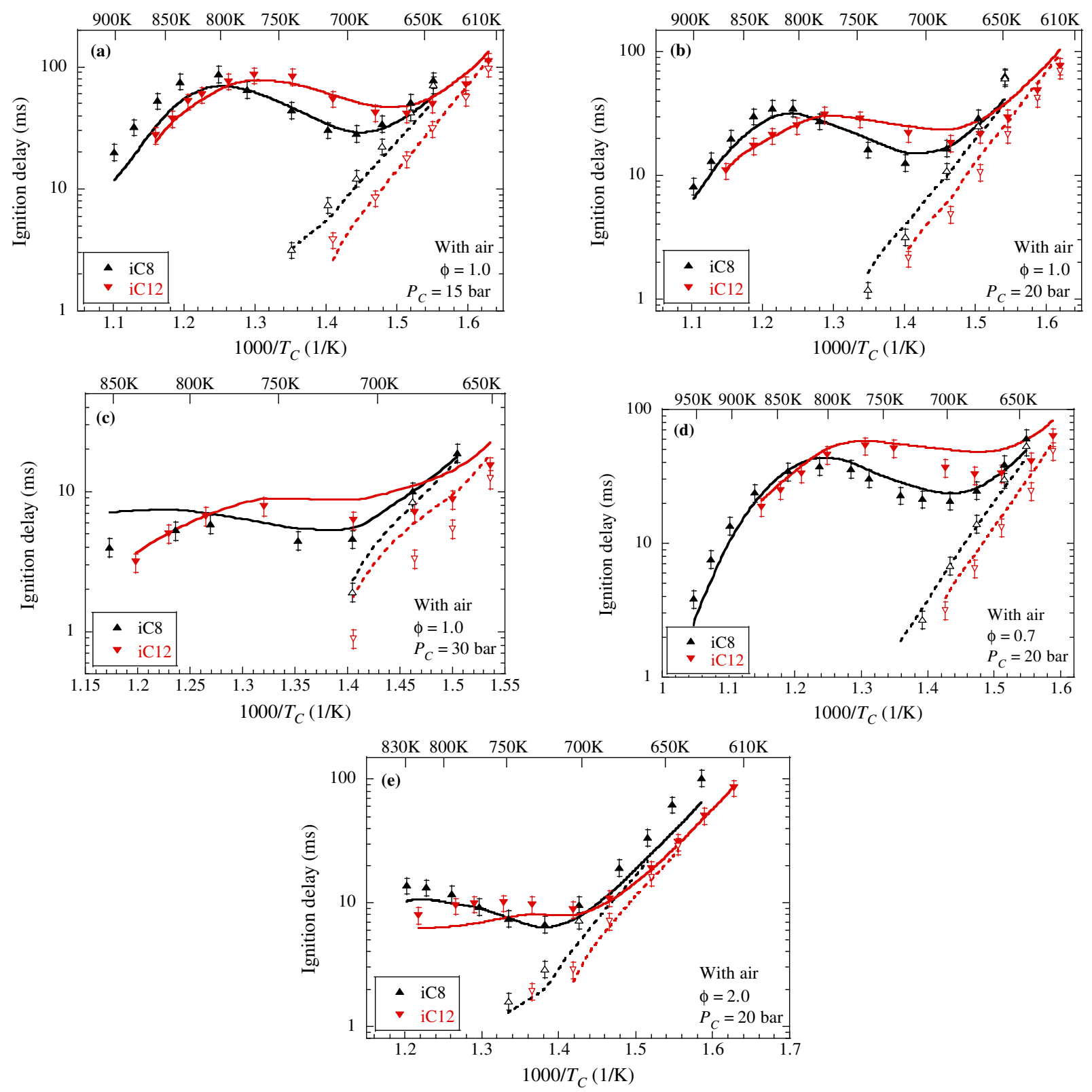

Figure 6: Experimental and simulated ignition delay times of $\mathrm{iC} 8 /$ air and iC12/air mixtures at different compressed pressures and equivalence ratios. (a) $P_{C}=15$ bar, $\phi=1.0$; (b) $P_{C}=20$ bar, $\phi=1.0$; (c) $P_{C}=30$ bar, $\phi=1.0$; (d) $P_{C}=20$ bar, $\phi=0.7$; (e) $P_{C}=20$ bar, $\phi=2.0$. Experimental results are shown as symbols. Filled symbols: total ignition delay times. Open symbols: first-stage ignition delay times. Simulated results are presented as lines. Solid lines: total ignition delay times. Dashed lines: first-stage ignition delay times. 

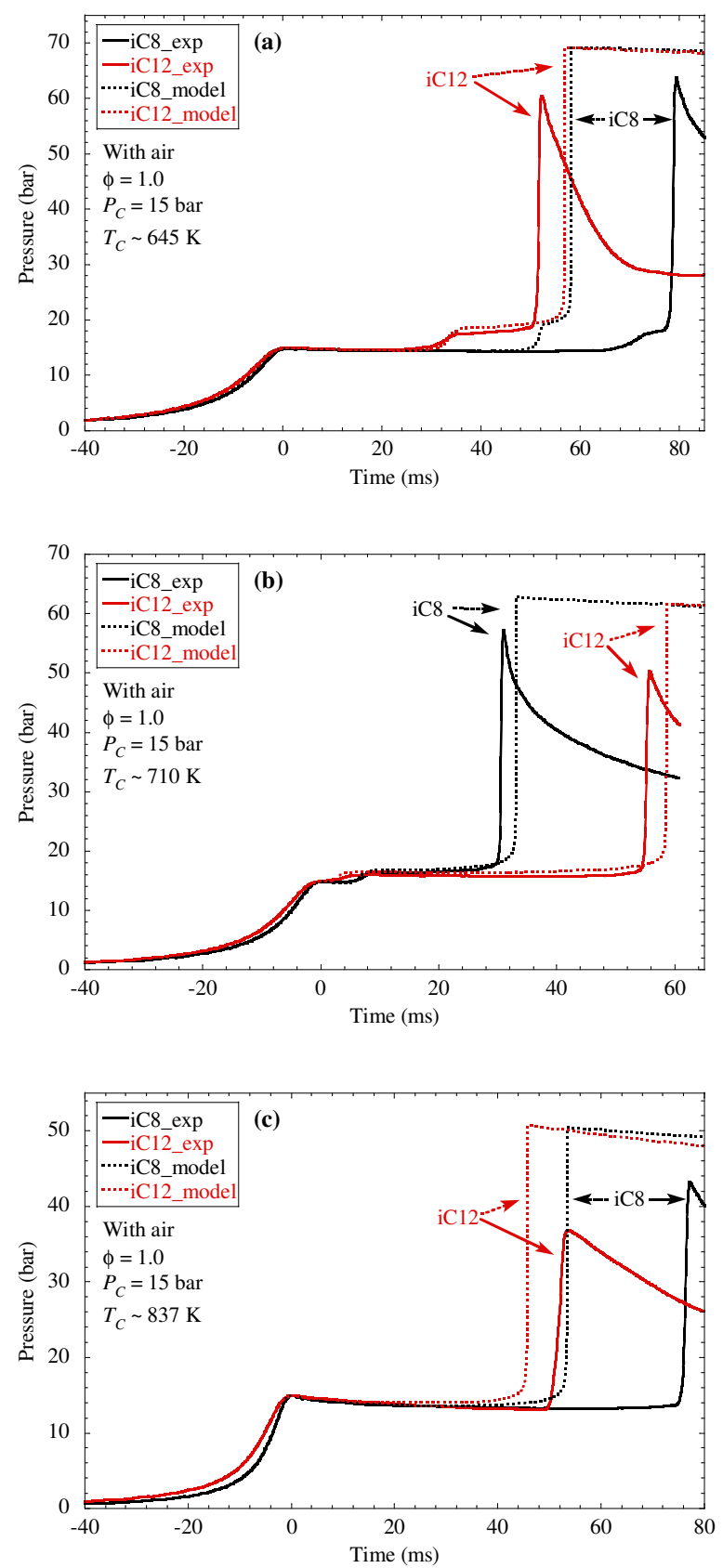

Figure 7: Experimental and simulated pressure trace comparison of stoichiometric iC8/air and iC12/air mixtures at $P_{C}=15$ bar and three representative compressed temperatures of (a) $T_{C} \sim 645 \mathrm{~K}$ (LTR); (b) $T_{C} \sim 710 \mathrm{~K}(\mathrm{NTC})$; and (c) $T_{C} \sim 837 \mathrm{~K}$ (ITR). 


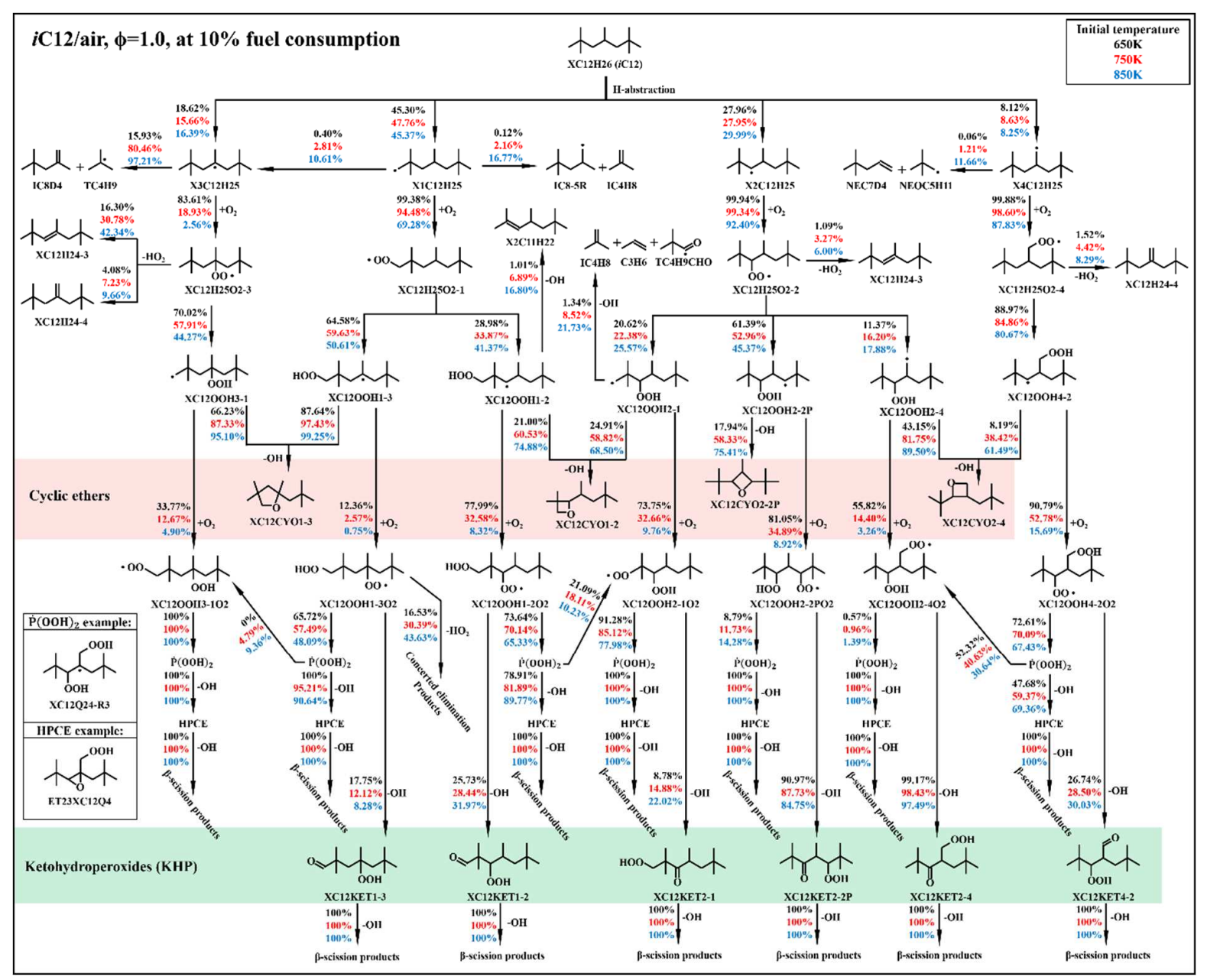

Figure 8: Major reaction pathways of $\mathrm{iC} 12$ based on CONV simulations at fuel consumption of $10 \%$, under $\phi=1.0$ in air, initial pressure of $20 \mathrm{bar}$, and initial temperatures of $650 \mathrm{~K}, 750 \mathrm{~K}$, and $850 \mathrm{~K}$. The colored number is the branching percent of each reaction at given initial temperature. 
(a) iC8/Air, $\phi=1.0,20$ bar

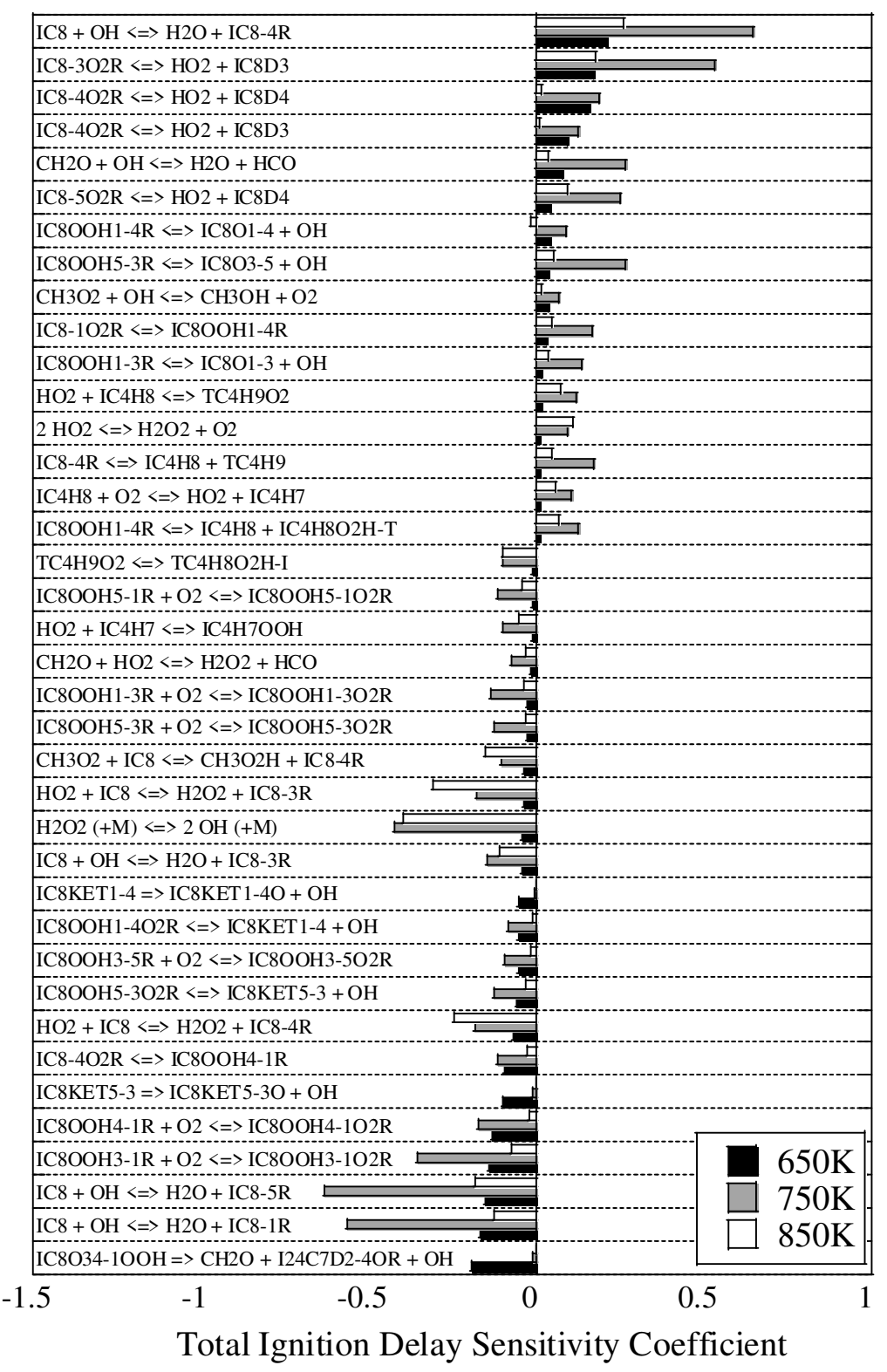


(b) iC12/Air, $\phi=1.0,20$ bar

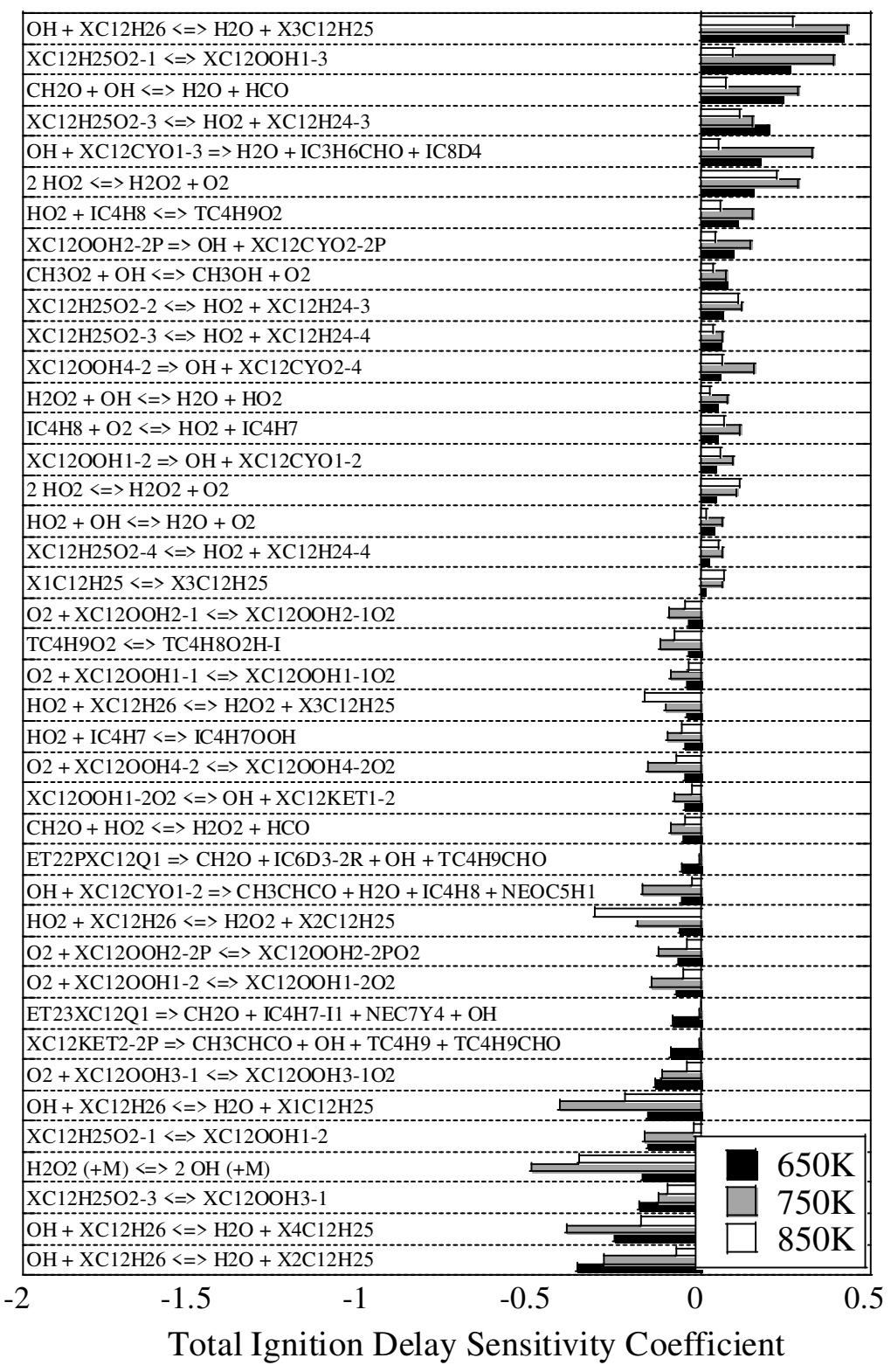

Figure 9: Brute force sensitivity coefficients for total ignition delay times of stoichiometric (a) iC8 and (b) iC12 in air based on CONV simulations at initial pressure of 20 bar with initial temperatures of 650 $\mathrm{K}, 750 \mathrm{~K}$, and $850 \mathrm{~K}$. 
(a) iC8/Air, $\phi=1.0,20$ bar, $650 \mathrm{~K}$

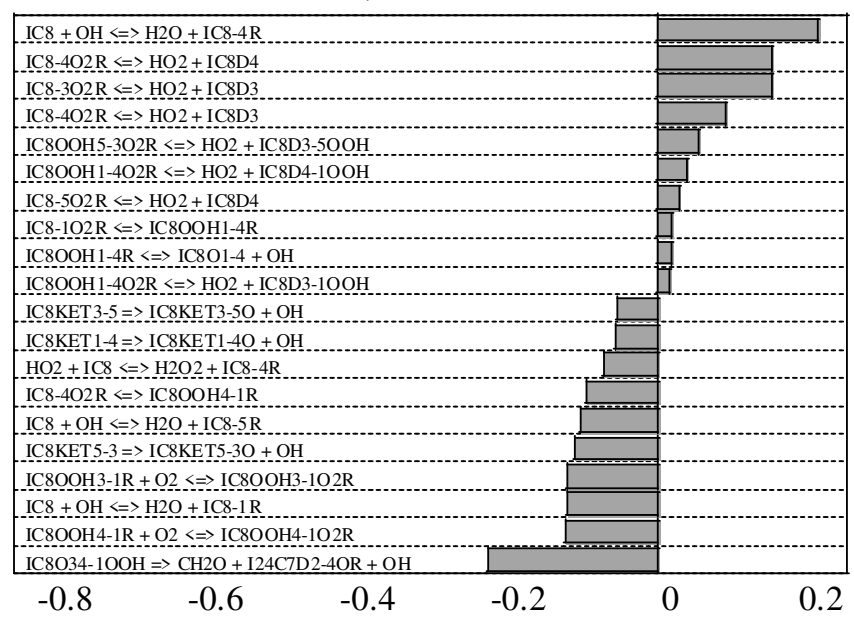

First-stage Ignition Delay Sensitivity Coefficient

(b) iC12/Air, $\phi=1.0,20$ bar, $650 \mathrm{~K}$

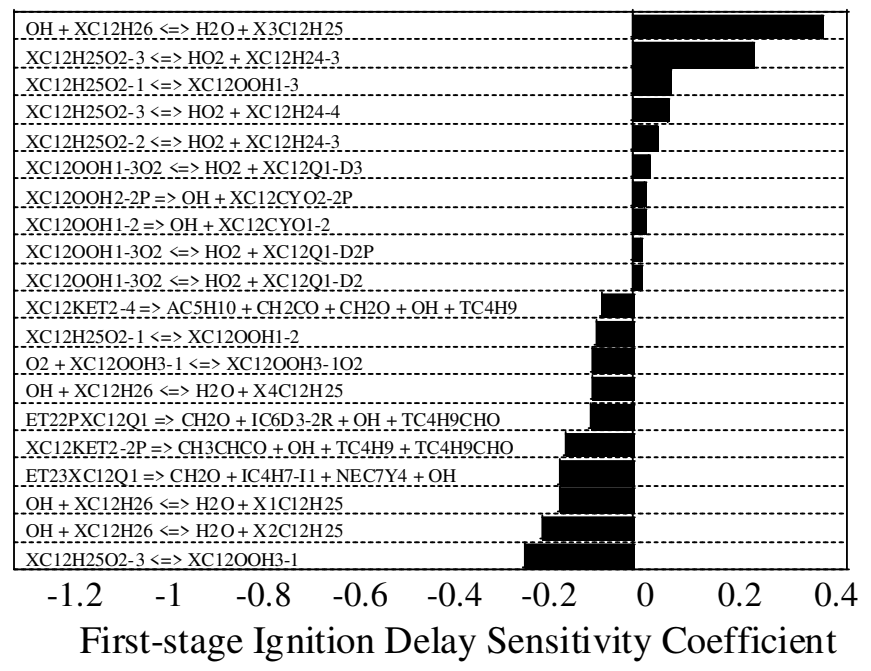

Figure 10: Brute force sensitivity coefficients for first-stage ignition delay times of (a) iC8 and (b) iC12 at $\phi=1.0 \mathrm{in}$ air, initial pressure of $20 \mathrm{bar}$, and initial temperature of $650 \mathrm{~K}$. 


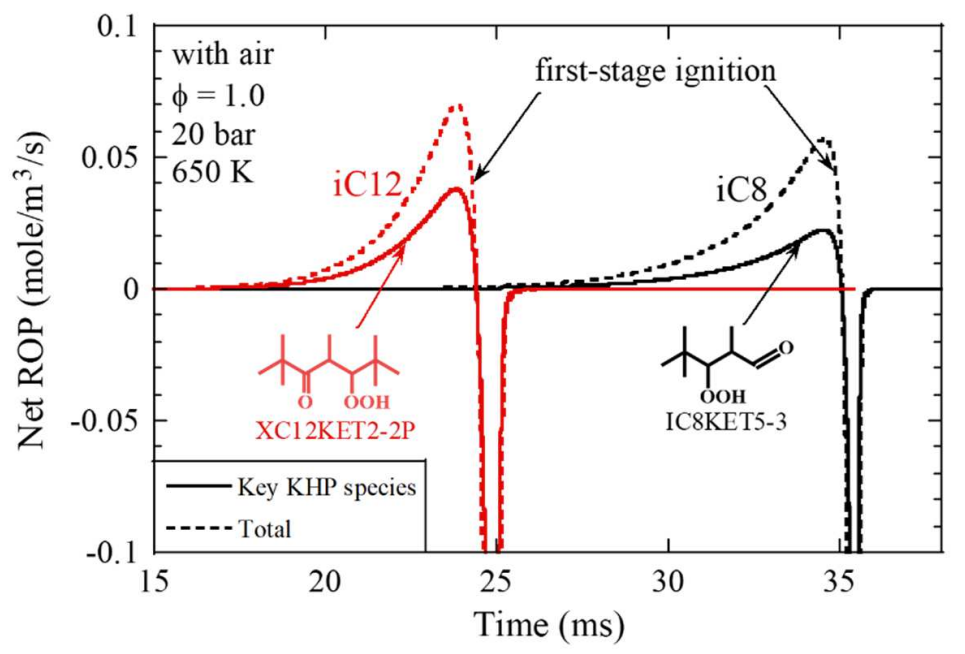

Figure 11: Comparison of net rate of production (ROP) of KHP for iC8 and iC12 at $\phi=1.0$ in air under initial conditions of 20 bar and $650 \mathrm{~K}$. Both the net ROP of the most dominant KHP species and the total net ROP for all KHP isomers are plotted.
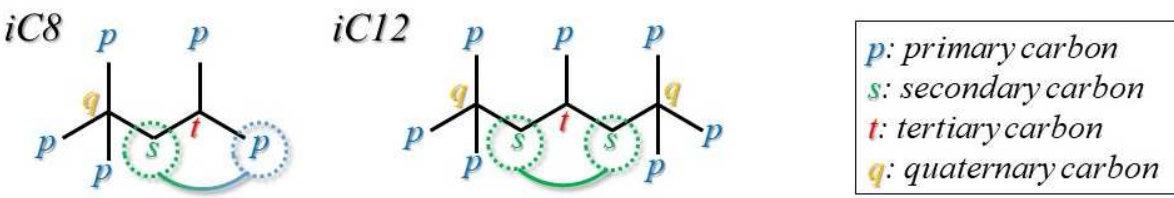

Written as a single step reaction in the model

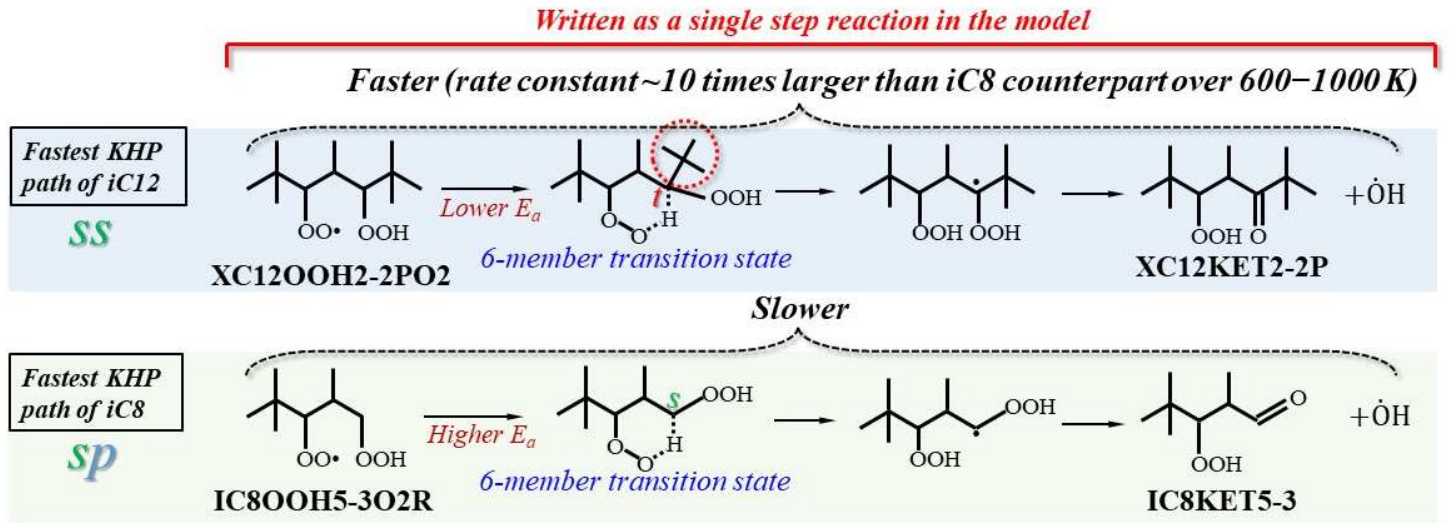

Figure 12: Molecular structure effect on low-temperature chain branching reactions forming KHP for $\mathrm{iC} 8$ and iC12. The symbols of $\mathrm{p}, \mathrm{s}, \mathrm{t}$, and q refer to the carbon types of primary, secondary, tertiary, and quaternary, respectively. 


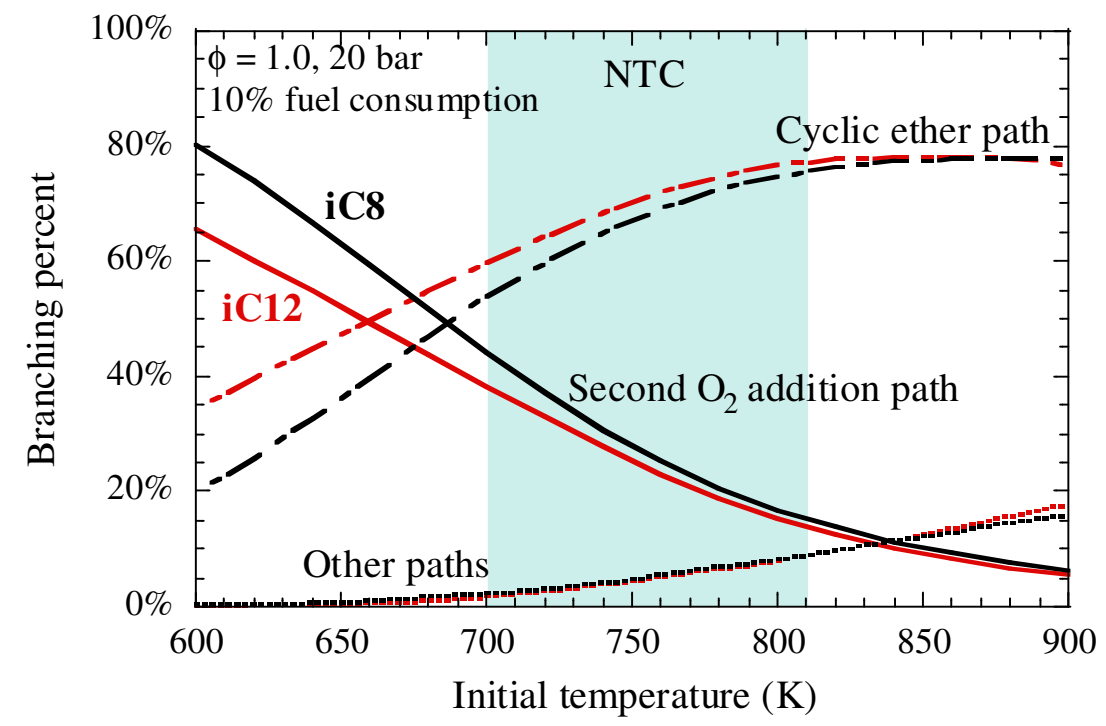

Figure 13: Branching percent of total $\mathrm{Q} O O H$ entering each pathway as a function of initial temperature for stoichiometric iC8 and iC12 in air at initial pressure of 20 bar.

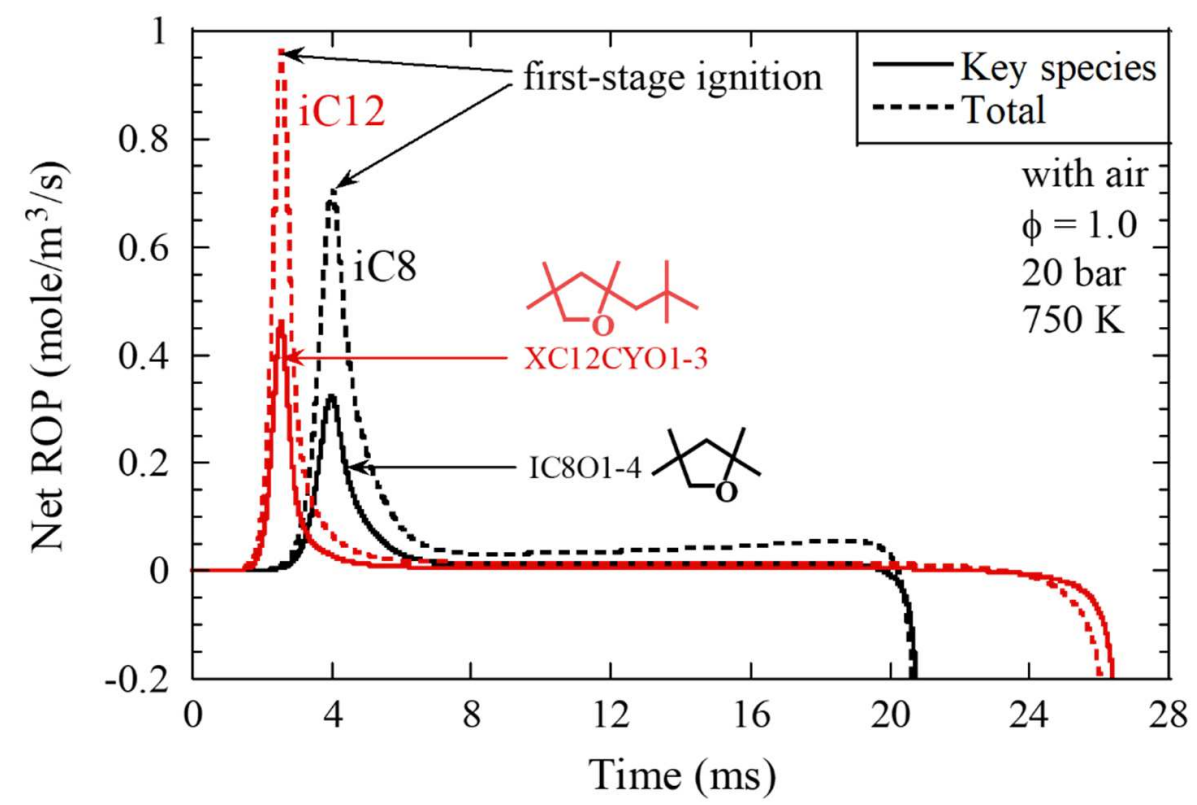

Figure 14: Comparison of net rate of production (ROP) of cyclic ethers for iC8 and iC12 at $\phi=1.0$ in air under initial conditions of 20 bar and $750 \mathrm{~K}$. Both the net ROP of the most dominant species and the total net ROP for all cyclic ethers are plotted. 
Tables:

Table 1: Summary of test conditions

\begin{tabular}{cccccc}
\hline \multirow{2}{*}{ Fuel type } & $\begin{array}{c}\text { Equivalence } \\
\text { ratio, } \phi\end{array}$ & $P_{C}($ bar $)$ & \multicolumn{3}{c}{ Molar proportions (\%) } \\
\cline { 4 - 6 } & 0.7 & 20 & 1.163 & 20.764 & 78.073 \\
iC8 & 1.0 & $15,20,30$ & 1.653 & 20.661 & 77.686 \\
& 2.0 & 20 & 3.252 & 20.325 & 76.423 \\
\hline \multirow{4}{*}{ iC12 } & 0.7 & 20 & 0.789 & 20.843 & 78.368 \\
& 1.0 & $15,20,30$ & 1.123 & 20.772 & 78.105 \\
& 2.0 & 20 & 2.221 & 20.542 & 77.237 \\
\hline
\end{tabular}

Table 2: Important low temperature reaction classes and the corrospond sources for reaction rates

\begin{tabular}{|c|c|c|}
\hline $\begin{array}{l}\text { Reaction } \\
\text { class index }\end{array}$ & Reaction Class & Sources \\
\hline$r_{2}$ & $\dot{\mathrm{R}}+\mathrm{O}_{2} \Leftrightarrow \mathrm{ROO}$ & Miyoshi [46] \\
\hline$r_{3}$ & $\mathrm{ROO} \Leftrightarrow \dot{\mathrm{QOOH}}$ & Villano et al. [47] \\
\hline$r_{13}, r_{14}$ & Concerted elimination of ROÓ and $\dot{O} O Q O O H$ & Villano et al. [47] \\
\hline$r_{11}$ & $\dot{\mathrm{Q} O O H} \Leftrightarrow$ Cyclic ethers $+\mathrm{OH}$ & Miyoshi [46], Villano et al. [48] \\
\hline$r_{15}$ & $\beta$-Q்OOH $\Leftrightarrow \mathrm{HO}_{2}+$ olefin & Villano et al. [48] \\
\hline$r_{4}$ & $\dot{\mathrm{QOOH}}+\mathrm{O}_{2} \Leftrightarrow \dot{\mathrm{O} O Q O O H}$ & Miyoshi [46] \\
\hline$r_{5}$ & ȮOQOOH $\Leftrightarrow$ Ketohydroperoxides (KHP) $+\dot{\mathrm{OH}}$ & Sharma et al. [49] \\
\hline$r_{7}$ & $\dot{\mathrm{O} O Q O O H} \Leftrightarrow \dot{\mathrm{P}}(\mathrm{OOH})_{2}$ & Villano et al. [47] \\
\hline$r_{16}, r_{17}$ & Decomposition of $\dot{\mathrm{Q} O O H}$ and $\dot{\mathrm{P}}(\mathrm{OOH})_{2}$ & Villano et al. [48] \\
\hline$r_{8}$ & $\dot{\mathrm{P}}(\mathrm{OOH})_{2} \Leftrightarrow$ Hydroperoxy cyclic ethers (HPCE) $+\dot{\mathrm{OH}}$ & Miyoshi [46], Villano et al. [48] \\
\hline$r_{6}, r_{9}$ & Decomposition of KHP and HPCE & See the text in Section 3.1 \\
\hline
\end{tabular}

